\title{
Cytoplasmic long noncoding RNAs are differentially regulated and translated during human neuronal differentiation
}

\author{
KATERINA DOUKA, ${ }^{1,2}$ ISABEL BIRDS, ${ }^{1,2}$ DAPENG WANG ${ }^{2}$ ANDREAS KOSTELETOS, ${ }^{1,2}$ SOPHIE CLAYTON, ${ }^{1,3}$ \\ ABIGAIL BYFORD, ${ }^{1,3}$ ELTON J.R. VASCONCELOS, ${ }^{2}$ MARY J. O'CONNELL, ${ }^{5}$ JIM DEUCHARS, ${ }^{2,3}$ \\ ADRIAN WHITEHOUSE, ${ }^{1,2,4}$ and JULIE L. ASPDEN ${ }^{1,2}$ \\ ${ }^{1}$ School of Molecular and Cellular Biology, Faculty of Biological Sciences, University of Leeds, Leeds LS2 9JT, United Kingdom \\ ${ }^{2}$ LeedsOmics, University of Leeds, Leeds LS2 9JT, United Kingdom \\ ${ }^{3}$ School of Biomedical Sciences, Faculty of Biological Sciences, University of Leeds, Leeds LS2 9JT, United Kingdom \\ ${ }^{4}$ Astbury Centre for Structural Molecular Biology, University of Leeds, Leeds LS2 9JT, United Kingdom \\ ${ }^{5}$ School of Life Sciences, Faculty of Medicine and Health Sciences, The University of Nottingham, Nottingham NG7 2UH, United Kingdom
}

\begin{abstract}
The expression of long noncoding RNAs is highly enriched in the human nervous system. However, the function of neuronal IncRNAs in the cytoplasm and their potential translation remains poorly understood. Here we performed Poly-Ribo-Seq to understand the interaction of IncRNAs with the translation machinery and the functional consequences during neuronal differentiation of human SH-SY5Y cells. We discovered 237 cytoplasmic IncRNAs up-regulated during early neuronal dif-

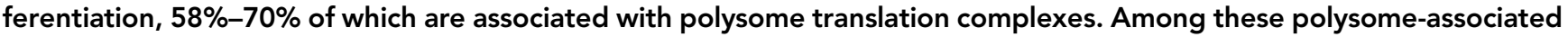
IncRNAs, we find $\mathbf{4 5}$ small ORFs to be actively translated, 17 specifically upon differentiation. Fifteen of 45 of the translated IncRNA-smORFs exhibit sequence conservation within Hominidea, suggesting they are under strong selective constraint in this clade. The profiling of publicly available data sets revealed that 8/45 of the translated IncRNAs are dynamically expressed during human brain development, and 22/45 are associated with cancers of the central nervous system. One translated IncRNA we discovered is LINC01116, which is induced upon differentiation and contains an 87 codon smORF exhibiting increased ribosome profiling signal upon differentiation. The resulting LINC01116 peptide localizes to neurites. Knockdown of LINC01116 results in a significant reduction of neurite length in differentiated cells, indicating it contributes to neuronal differentiation. Our findings indicate cytoplasmic IncRNAs interact with translation complexes, are a noncanonical source of novel peptides, and contribute to neuronal function and disease. Specifically, we demonstrate a novel functional role for LINC01116 during human neuronal differentiation.
\end{abstract}

Keywords: IncRNA; neuronal differentiation; polysome; ribosome profiling; translation

\section{INTRODUCTION}

Long noncoding RNAs (IncRNAs) are >200 nt in length and thought to lack the ability to encode proteins. LncRNAs are less conserved, yet more tissue- and developmentalstage-specific than mRNAs (Tsagakis et al. 2020). Early work indicated that the majority of IncRNAs were predominantly nuclear and localized to chromatin (Derrien et al. 2012; Djebali et al. 2012). However, it has become increasingly clear that many IncRNAs are exported to the cytoplasm, and recent estimates are that $\sim 54 \%$ of IncRNAs are detected in the cytoplasm (Carlevaro-Fita et al.

Corresponding author: j.aspden@leeds.ac.uk

Article is online at http://www.rnajournal.org/cgi/doi/10.1261/rna. 078782.121. Freely available online through the RNA Open Access option.
2016). Although many IncRNAs have been found to bind proteins, biological functions have only been determined for a relatively small number of IncRNAs.

Several IncRNAs have been found to play key roles in development and differentiation; for example, Inc-31 during myoblast differentiation (Dimartino et al. 2018). They are particularly enriched in the nervous system of Drosophila melanogaster, mouse and human. It is estimated that $\sim 40 \%$ of human IncRNAs are specifically expressed in the brain (Derrien et al. 2012), where they display precise spatiotemporal expression profiles (Ponting et al. 2009). A subset of nuclear neuronal IncRNAs have been found

(C) 2021 Douka et al. This article, published in RNA, is available under a Creative Commons License (Attribution 4.0 International), as described at http://creativecommons.org/licenses/by/4.0/. 
to regulate neuronal differentiation in mouse and human (Chodroff et al. 2010; Lin et al. 2014; Winzi 2018; Carelli et al. 2019). However, only a small number of cytoplasmic IncRNAs have had their biological and molecular functions elucidated. These include IncRNAs found to associate with translation complexes (Carrieri et al. 2012) and to have specific cytoplasmic functions in posttranscriptional gene regulation. For example, BACE1-AS transcript, which is significantly up-regulated in the brains of Alzheimer's disease patients, base-pairs with beta-secretase-1 (BACE1) mRNA, stabilizing it (Faghihi et al. 2010), whereas BC200 represses translation initiation in dendrites by disrupting the formation of preinitiation $48 \mathrm{~S}$ complexes (Wang et al. 2002). Together these studies indicate that there may be many IncRNAs present in the cytoplasm, potentially playing important roles during neuronal development and differentiation that are yet to be discovered.

Ribosome profiling (Ribo-Seq) in a range of organisms and tissue types has revealed the translation of a variety of noncanonical ORFs, including small ORFs (smORFs) $<100$ codons in length from within IncRNAs (Guo et al. 2010; Ingolia et al. 2013; Aspden et al. 2014; Duncan and Mata 2014; Blair et al. 2017; Fujii et al. 2017; Rodriguez et al. 2019). Although these translation events remain controversial, it is clear that IncRNAs can interact with the translation machinery (Ruiz-Orera and Alba 2019). Limited ribosome profiling signal found on smORFs might be the result of sporadic binding of a single ribosome and may not necessarily correspond to active translation (Patraquim et al. 2020). We previously developed Poly-Ribo-Seq to distinguish those IncRNAs that are bound by multiple ribosomes, and therefore actively translated, from nonspecific background signal (Aspden et al. 2014). A small but growing number of smORF peptides translated from IncRNAs have been found to exhibit cellular and organismal functions (Pueyo and Couso 2008; Magny et al. 2013; Anderson et al. 2015; Chen et al. 2020; Spencer et al. 2020; Wang et al. 2020). Therefore, the identification of genuine smORF translation events from IncRNAs by ribosome profiling is an important first step in assessing the wider importance of these smORF peptides. To date, a robust assessment of IncRNA translation in the context of neuronal differentiation, where IncRNA expression is enriched, has been missing.

Given (i) the large number of IncRNAs enriched in the human central nervous system, (ii) recently revealed IncRNA roles in differentiation, and (iii) evidence of translation of IncRNA to produce small peptides, we reasoned that IncRNAs may functionally interact with polysomes and be translated during neuronal differentiation. This work aimed to probe the dynamic interactions of IncRNAs with the translation machinery and identify actively translated cytoplasmic IncRNAs during early neuronal differentiation. This will be important to understand the biological role of cytoplasmic IncRNAs and to identify novel peptides with potentially biological and medically important functions.

Here, we have performed Poly-Ribo-Seq (Aspden et al. 2014), an adaptation of ribosome profiling, to determine the population of IncRNAs present in the cytoplasm, assess their interaction with the translation machinery, and establish which IncRNAs are translated. We used the human neuronal cell line SH-SY5Y to provide a model of neuronal differentiation and to generate sufficient material for Poly-Ribo-Seq. We followed up our transcriptome wide analysis probing a subset of candidate IncRNAs in more detail in terms of their enrichment in the cytoplasm, precise association with translation complexes, and ORF tagging experiments. For the translated IncRNAs we identified, we have assessed their conservation and their importance to neuronal development and disease using previously published data sets. For one translated candidate IncRNA, LINC01116, we characterized its functional contribution to neuronal differentiation.

\section{RESULTS}

\section{Differentiation of SH-SY5Y cells with retinoic acid results in reduced translation levels}

To dissect the importance of cytoplasmic IncRNAs and their ribosome associations in early neuronal differentiation, we profiled the differentiation of SH-SY5Y cells with retinoic acid (RA) for $3 \mathrm{~d}$. This treatment results in neuronal differentiation as indicated by neurite elongation, which can be seen by immunostaining for neuronal $\beta$ III-tubulin (TuJ1) (Supplemental Fig. 1A), and quantification of neurite length reveals significant elongation upon RA treatment (Supplemental Fig. 1B). There is also increased expression of neuronal markers: more cells express c-Fos upon differentiation (Supplemental Fig. 1C,D). There is a concomitant reduction in cell proliferation, as seen by the reduced number of Ki67+ cells (Supplemental Fig. $1 \mathrm{E}, \mathrm{F})$. Together this data indicates that our RA treatment of SH-SY5Y cells leads to neuronal differentiation.

To determine if this RA-induced differentiation of the $\mathrm{SH}$ SY5Y model will be suitable to study translational dynamics, the translational output of these cells was assessed by polysome profiles (Supplemental Fig. 2A). This revealed that differentiation results in down-regulation of global translation. Quantification of translation complexes across the sucrose gradients indicates that levels of polysomes are reduced with respect to 805 monosomes, resulting in a reduced polysome to monosome ratio (Supplemental Fig. 2B). This down-regulation of translation is accompanied by a shift of ribosomal protein (RP) mRNAs from polysomes to monosomes: for example, RPL26 mRNA (Supplemental Fig. 2C), RPS28 (Supplemental Fig. 2D), and RPL37 (Supplemental Fig. 2E), as measured by RT-qPCR across gradient fractions. This reduced synthesis 
of RPs has previously been reported during neuronal differentiation (Blair et al. 2017; Chau et al. 2018). Together these data indicate that the model of RA-induced neuronal differentiation of $\mathrm{SH}-\mathrm{SY} 5 \mathrm{Y}$ cells, with dynamically regulated translation, provides an ideal system in which to study cytoplasmic RNAs, their interaction with the translation machinery, and contribution to neuronal differentiation.

\section{Cytoplasmic IncRNA expression is regulated during neuronal differentiation}

To profile RNA, ribosome association, and translational changes upon differentiation, we used Poly-Ribo-Seq (Aspden et al. 2014) with some minor modifications to adapt to human neuronal cells (Fig. 1A). This adaptation of ribosome profiling (Ribo-Seq) can detect which RNAs are (a) cytoplasmic, (b) polysome-associated, and (c) translated (Fig. 1A). We sequenced (i) poly(A) selected cytoplasmic RNA, "Total" RNA-seq, (ii) polysome-associated poly(A) RNAs, "Polysome" RNA-seq, and (iii) ribosomal footprints, RiboSeq, from "Control" and "RA" differentiated cells (Fig. 1A).

To determine which IncRNAs are expressed, present in the cytoplasm, and regulated during differentiation, we first analyzed total RNA-seq (Supplemental Fig. 3A). PCA revealed that RA treated samples cluster separately from Control samples and biological replicates generally cluster together (Supplemental Fig. 3B). We detected large numbers of IncRNAs expressed and present (i.e., have RPKMs $\geq$ 1) in the cytoplasm. In the Control conditions there were 801 IncRNA genes expressed in the cytoplasm and 916 IncRNA genes in differentiated cells. To understand the potential role and regulation of cytoplasmic IncRNAs during neuronal differentiation, we performed differential expression analysis between Control and RA samples at the gene level. We observed 178 IncRNA genes up-regulated and 100 down-regulated during differentiation in the total cytoplasm (Supplemental Fig. 3C). We also performed differential expression analysis at the gene level for Polysomal RNA-seq samples (Fig. 1A). Within the Polysomes, we identified 237 IncRNA genes that were up-regulated during differentiation while only 82 were down-regulated (Fig. 1B). Comparing the IncRNAs differentially regulated in Total and Polysomes populations, the majority, i.e., $71 \%$ of the up-regulated $(126 / 178)$ and $71 \%$ of the down-regulated IncRNAs (58/82) found in Polysomes were also found in Total (Supplemental Fig. $3 D, E)$. Significant induction of specific IncRNAs during differentiation, such as DLGAP1-AS2, is suggestive of a regulatory role during neuronal differentiation (Supplemental Fig. 3F). An assessment of the types of IncRNA regulated during neuronal differentiation revealed that the majority are either intergenic or antisense IncRNAs, 216/237 for up-regulated (Fig. 1C) and 74/82 for down-regulated (Fig. 1D). In summary, neuronal differentiation results in differential expression of $\sim 300$ IncRNAs within the cytoplasm.
We validated the differentiation-induced changes in a subset of seven IncRNAs (Supplemental Fig. 4A). By RT-qPCR the expressions of $6 / 7$ candidate IncRNAs were significantly $(P<0.05$ : SNAP25-AS1, LINC001116; $P$ <0.01: ACE254633.1, DLGAP1-AS2, DLGAP1-AS1, LINC02143) up-regulated upon differentiation, as was determined by RNA-seq analysis. Fold-changes were highly correlative between RNA-seq and RT-qPCR (Supplemental Fig. 4B). To enable us to focus on IncRNAs with potential neuronal functions, we selected candidate IncRNAs that exhibited the highest fold increase in levels upon differentiation. To understand whether our candidate IncRNAs are genuine cytoplasmic IncRNAs or whether their cytoplasmic population represents a small minority, we assessed their subcellular distribution. The majority of these candidate IncRNAs (6/7) are specifically enriched in the cytoplasm, rather than the nucleus (Fig. 1E; Supplemental Fig. 4C), in contrast to the known nuclear IncRNA XIST. Together these data indicate that $\sim 900$ IncRNAs are localized to the cytoplasm; the majority of these are likely enriched in the cytoplasm and $\sim 30 \%$ of these cytoplasmic IncRNAs are dynamically expressed during neuronal differentiation.

\section{Association of IncRNAs with polysomes during neuronal differentiation}

To determine the propensity of cytoplasmic IncRNAs to associate with translation complexes and how this is regulated during differentiation, we compared IncRNAs levels between the whole cytoplasm (Total-RNA-seq) and the polysomes (Polysome-RNA-seq). This analysis indicated that the vast majority of IncRNAs (Control: $98 \%$ and RA: 99\%) are neither polysome enriched nor depleted (Fig. $2 A, B)$. This suggests that most IncRNAs are not actively targeted to polysomes but present in translation complexes. A small number (Control: 12 and RA: 10) of IncRNAs are specifically depleted from the polysomes (Fig. 2A,B). This suggests that the roles these IncRNAs play are likely elsewhere in the cytoplasm and not directly connected to translation. Nine of 12 depleted in Control are not depleted upon differentiation, indicating their polysome association is regulated during differentiation (Supplemental Fig. 5A). There is a smaller proportion of antisense IncRNAs within these polysome-depleted populations (Supplemental Fig. 5B,C) as compared to the proportion displayed by those IncRNAs differentially expressed during differentiation (Fig. 1C,D). This may indicate that antisense IncRNAs are more likely to be present in polysomes, and their role in the polysomes could be linked to their antisense characteristics.

To understand the precise nature of the association of our candidate IncRNAs with translation complexes, their distribution was profiled across sucrose gradient fractions. This confirmed that these IncRNAs are found to associate with polysome complexes within the cytoplasm but also 


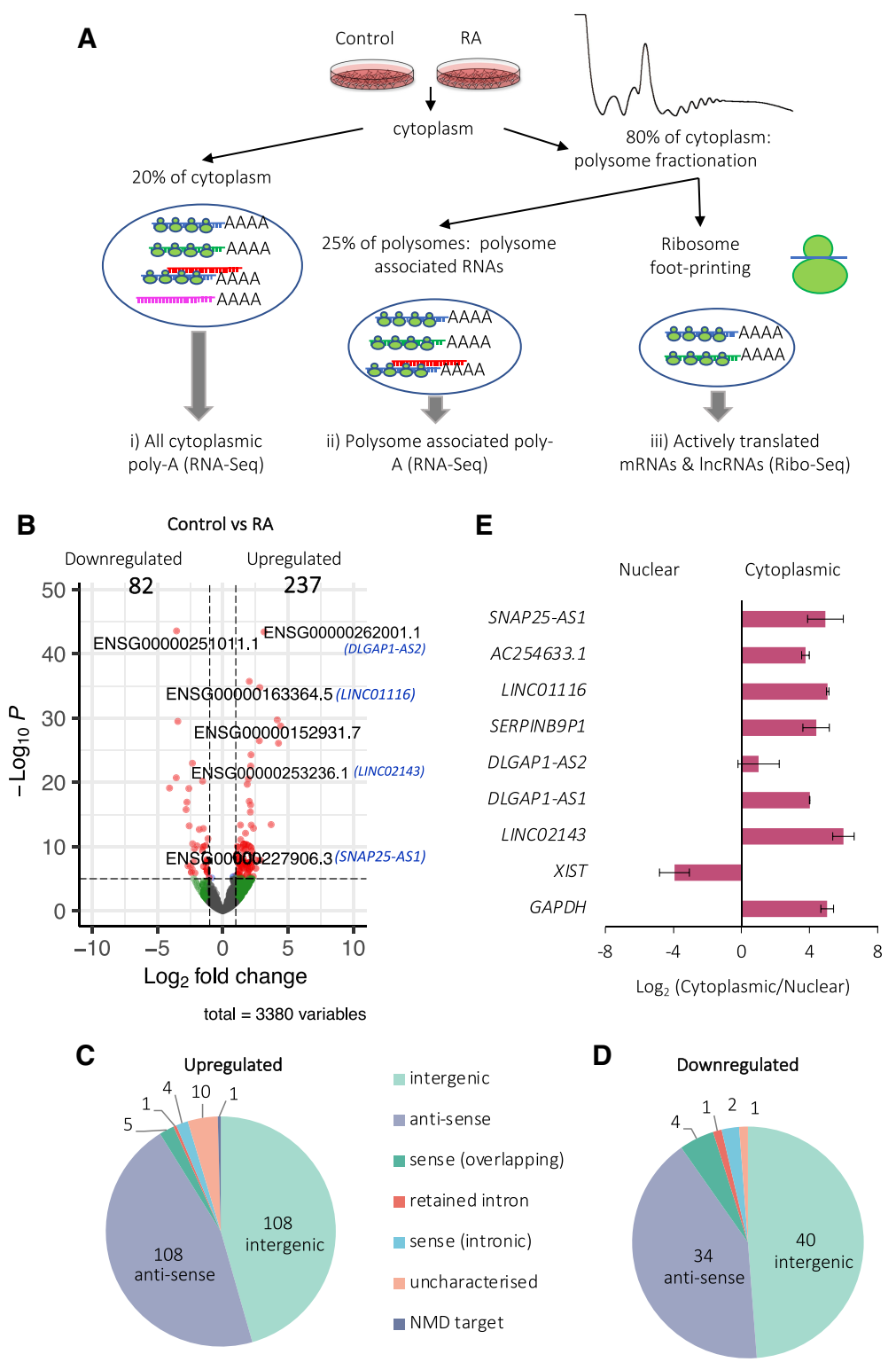

FIGURE 1. Cytoplasmic IncRNA expression is regulated during neuronal differentiation. (A) Schematic of Poly-Ribo-Seq, with three levels of analysis: (i) total cytoplasmic, (ii) polysome-associated, and (iii) translated IncRNAs. (B) Volcano plot of differential expression analysis of polysome-associated IncRNAs (labeled by genelDs and names for candidate IncRNAs) between Control and RA populations. Two hundred and thirty-seven IncRNAs are up-regulated during differentiation and 82 down-regulated $\left(\log _{2}\right.$ fold-change cutoff $\left.=1, p^{\text {adj }}<0.05\right)$. Pie chart of types of IncRNAs $(C)$ up-regulated and $(D)$ down-regulated upon differentiation (intergenic; antisense; sense-overlapping; retained intron; sense-intronic; uncharacterized; NMD target). (E) LncRNAs of interest that are induced are specifically localized to cytoplasm as shown by subcellular fractionation RT-GPCR. XIST IncRNA was used as a nuclear and GAPDH mRNA as a cytoplasmic positive control $(n=3$, SE is plotted, Student's t-test, $n=3$, $P>0.05)$. the nucleus (Fig. 1E). LINC02143 was mainly found in monosomes (80S) and small polysomes (two to three ribosomes) (Fig. 2C). DLGAP1-AS1 was also found to associate with small polysomes (two to four ribosomes), as well as ribosomal subunits and $80 \mathrm{~S}$ monosomes (Fig. 2D). Therefore, both LINC02143 and DLGAP1-AS1 could either be translated or regulate translation.

Another IncRNA whose levels significantly increase during differentiation is LINC01116 (Fig. 1B; Supplemental Fig. 4A), which has previously been shown to be involved in the progression of glioblastoma (GBM) (Brodie et al. 2017). LINC01116 is enriched in the cytoplasm (Fig. 1E), detected at high levels in the $80 \mathrm{~S}$ (monosome) fraction and in small and medium polysomes (two to seven ribosomes) (Fig. 2E). Upon differentiation there is an increase in the amount of LINC01116 present in disomes, compared to Control. This is consistent with the up-regulation of the LINC01116 transcript in the polysomes detected by RNA-seq, indicating a likely functional interaction of LINC01116 with polysomes during differentiation. In fact, the majority of the LINC01116 transcript was found to associate with polysomes in both undifferentiated cells (Control-66\%) and upon differentiation (RA-57\%), suggesting it is could either be translated or associating with translation complexes (Fig. 2E). Overall, these data indicate that the majority of cytoplasmic IncRNAs are polysome-associated but not enriched. Comparing the differentiation-induced IncRNA changes between Total and Polysome populations, as well as specific candidate IncRNAs, also suggests that polysome association is dynamic during differentiation. reveals the precise translation complexes they interact with in terms of ribosomal subunits, $80 \mathrm{~S}$ monosomes, and different polysomes. We first profiled LINC02143, which is induced $>22$-fold during differentiation (Supplemental Fig. $4 \mathrm{~A})$ and highly enriched in the cytoplasm compared to

\section{Translation of IncRNA-smORFs during differentiation}

To better understand the association of IncRNAs with polysome complexes and their potential translation, we analyzed our third and final data set, ribosome footprinting 
A

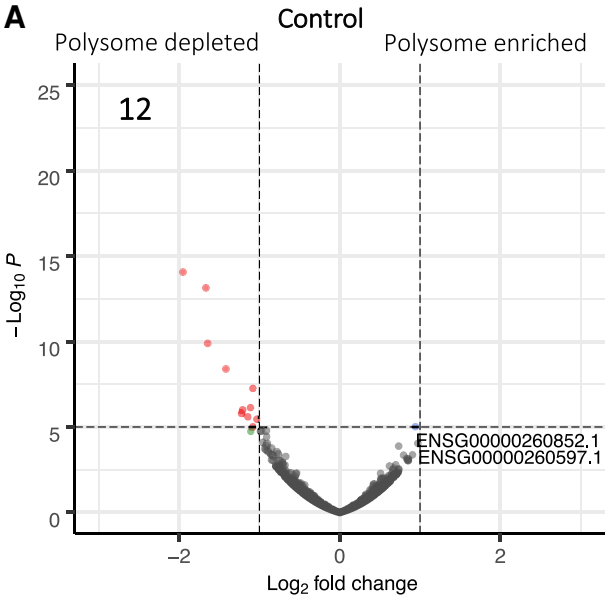

B

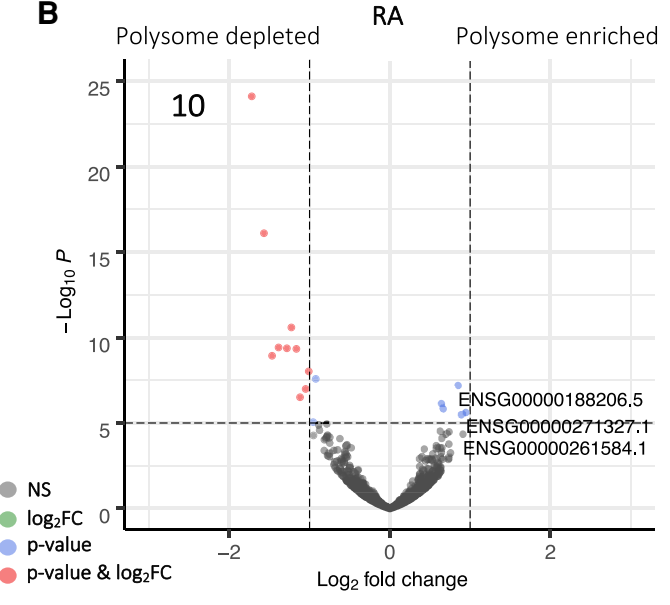

C

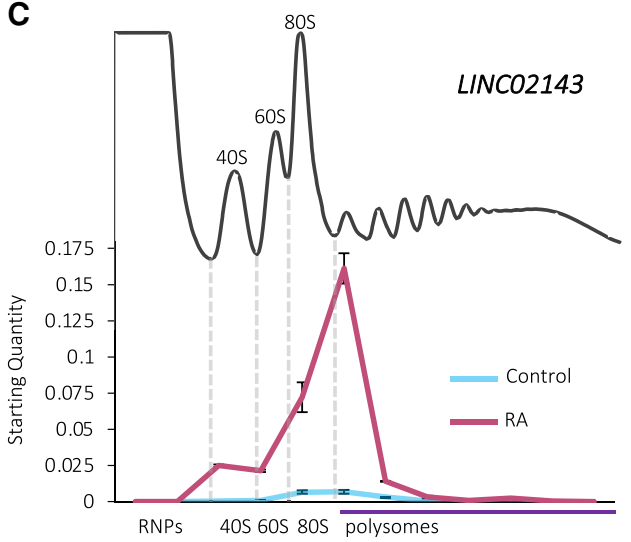

D

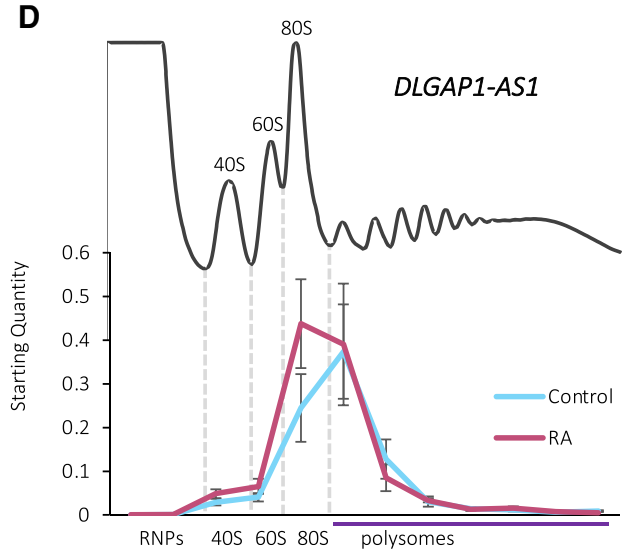

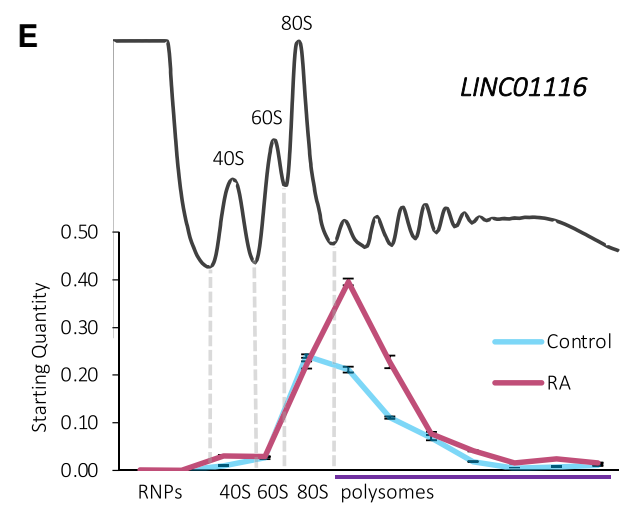

FIGURE 2. Association of IncRNAs polysomes during neuronal differentiation. Volcano plots displaying the significantly differentially localized IncRNAs (labeled by their geneIDs) between Total and Polysome populations in (A) Control and (B) RA, with $\log _{2}$ fold-change cutoff $=1$, $P^{\text {adj }}<$ 0.05. (C-E) RT-qPCR of IncRNAs across sucrose gradient fractions ( $n=3$, SE is plotted). (C) LINC02143 is found in $80 S$ and small polysome fractions during differentiation. Five percent of the transcript is detected in $80 \mathrm{~S}$ (monosome) fractions and $66 \%$ in small polysome complexes. (D) DLGAP1-AS2 is found in $80 \mathrm{~S}$ and small polysome fractions both in control and RA treated cells. On average, $63 \%$ of the transcript is detected in the polysome fractions in Control and 49\% upon differentiation. (E) LINC01116 is found in 80S and two to seven polysome fractions both in control and RA treated cells. On average, $66 \%$ of the LINC01116 transcript is detected in the polysome fractions in Control and $57 \%$ upon differentiation.

from our Poly-Ribo-Seq experiments (Fig. 1A, actively translated mRNAs and IncRNAs). Triplet periodicity analysis reveals good framing, specifically at footprint lengths of
31 and 33 nt (Supplemental Fig. 6A; Supplemental Table 1). On average, $95 \%$ of ribosome footprints mapped to CDSs, while in RNA-seq this was only 53\% (Supplemental 
Fig. 6B). Together, these attributes indicate that our RiboSeq quality is high and represents genuine translation events. Since 31 and 33 nt reads exhibited high triplet periodicity, they were selected for downstream analysis of translation events to identify ORFs that were translated (Fig. 3A). Overall, we are able to detect the translation of both canonical protein-coding ORFs and noncanonical ORFs, including upstream (uORFs) and downstream ORFs (dORFs), in both Control and RA conditions (Table 1).

We analyzed our Ribo-Seq data in order to determine if any of the polysome-associated IncRNAs are engaged with ribosomes and translated. Using our pipeline (Fig. $3 A$ ), we detected the translation of 45 small ORFs within IncRNAs (IncRNA-smORFs), 28 in Control and 23 during differentiation; six of these were translated in both conditions (Fig. 3B). Only two of these IncRNA-smORFs have previously been characterized, CRNDEP (Szafron et al. 2015) and HAND2-AS1 (van Heesch et al. 2019); the remaining 43 represent novel ORFs. The level at which these IncRNA-smORFs are translated was assessed by determining their translational efficiencies (TEs), the number of ribosome footprints relative to RNA abundance. The TEs of translated IncRNA-smORFs were similar to those measured for protein-coding ORFs (Fig. 3C), providing further evidence that these are genuine translation events, whereas the dORFs that we detect are translated at much lower efficiencies (Fig. 3C). This is likely to be because ribosomes would have to reinitiate after the main ORF, which would occur at a lower efficiency. As an additional assessment of whether the translation of IncRNA-smORFs represent genuine translation events, we compared the pattern of ribosome footprints across the smORFs with protein-coding CDSs (Supplemental Fig. 6C-F). In both Control and RA conditions, metagene plots show that the distribution of footprints is very similar between IncRNA-smORFs (Supplemental Fig. 6C,D) and protein-coding CDSs (Supplemental Fig. 6E,F), specifically around start and stop codons. There is a substantial drop-off of footprints at the stop codon for both, indicative of genuine translation events. Together, our Ribo-Seq analysis reveals that a subset of polysome-associated IncRNAs is translated.

To better understand these translated IncRNA-smORFs, we profiled their features. Analysis of the 45 translated smORFs from IncRNAs shows that they are all $<300$ aa in length with a median size of 60 aa (Fig. 3D) (56 aa in Control and 64 aa in RA: Supplemental Fig. 7A). Previous analysis has indicated that Drosophila smORF peptides exhibit specific amino acid usage, indicating that they are genuine proteins and show a propensity to form transmembrane $\alpha$-helices (Aspden et al. 2014). Therefore, we profiled the amino acid composition of our IncRNAssmORFs, uORFs, and dORFs compared to protein-coding ORFs and expected by chance frequencies (Supplemental Fig. 7B). IncRNA-smORFs exhibit similar frequencies to known protein-coding ORFs. Specifically, smORFs possess lower than expected arginine levels, but not as low as known protein-coding ORFs. Amino acid usage does not suggest that any smORF groups have a propensity to form transmembrane $\alpha$-helices.

From within the set of IncRNAs we identified as induced during differentiation (RNA-seq), several contained translated smORFs (Ribo-Seq). One of these is LINC01116, which contains a 71-codon smORF detected as translated by our Ribo-Seq data. The ribosome profiling signal is substantially higher upon differentiation (Fig. 3E; Supplemental Fig. 7C), mainly as a result of increased IncRNA transcript abundance. Overall, $\sim 80 \%$ of reads that map to this ORF are in frame 2, whereas outside this ORF, the few reads mapping to the remaining IncRNA sequence are far more equally distributed between the three possible frames (Fig. 3E). Such robust framing is highly indicative of genuine translation of this specific smORF, from within the LINC01116 IncRNA. Together, analysis of our Ribo-Seq data has led to the discovery of 43 novel IncRNA-smORFs with robust indicators of translation.

\section{Peptide synthesis from smORFs in IncRNAs during differentiation}

Our pipeline is highly stringent, that is, there are many additional ORFs that display good framing but do not pass our thresholds for numbers of footprinting reads or exhibit background signals in the rest of the IncRNA. Therefore, we are confident these translation events are taking place. To validate peptide synthesis from these translation events, we have taken two complementary strategies: mass spectrometry analysis and transfection of ORF tagging constructs. Analysis of previously published mass spectrometry data sets from SH-SY5Y cells (undifferentiated and RA-treated) (Murillo et al. 2018; Brenig et al. 2020) supports the production of peptides from eight IncRNAsmORFs (four Control and four RA) (Fig. 4A). This relatively low level of support is to be expected, given the small size of these peptides and therefore the reduced chance of producing peptides >8aa from digestion for mass spectrometry detection (Saghatelian and Couso 2015). Overall mass spectrometry supports the peptide synthesis from $18 \%$ of IncRNA-smORFs detected by Poly-Ribo-Seq.

To validate translation of our IncRNA-smORFs that were not identified in previous mass spectrometry but were detected as translated by our Poly-Ribo-Seq analyses, we used a transfection tagging approach. We cloned the IncRNA sequence $5^{\prime}$ of the putative ORF, termed the $5^{\prime}-U T R$, and the smORFs, without its stop codon, with a carboxy-terminal 3x FLAG tag (Fig. 4B). The FLAG tag did not have its own start codon, so any FLAG signal is the result of translation from the IncRNA-smORF. Two candidate IncRNA-smORFs were selected that did not have mass spectrometry support: LINC000478 and LINC01116. A 37 codon smORF was detected as translated by our Poly- 
A

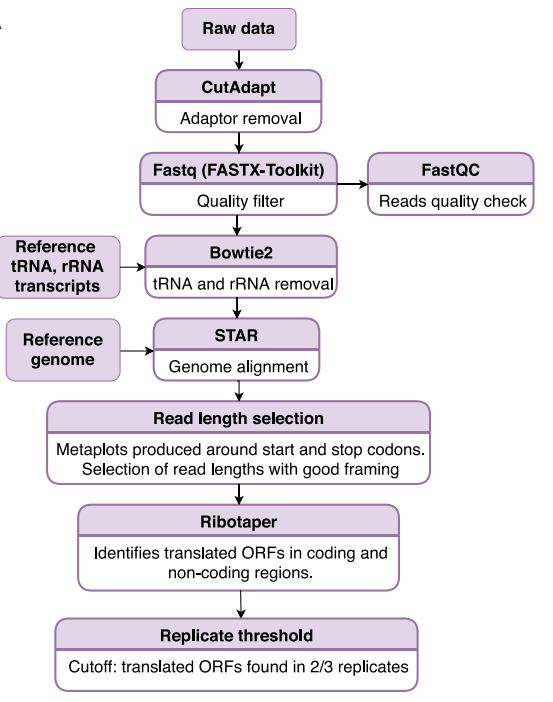

C

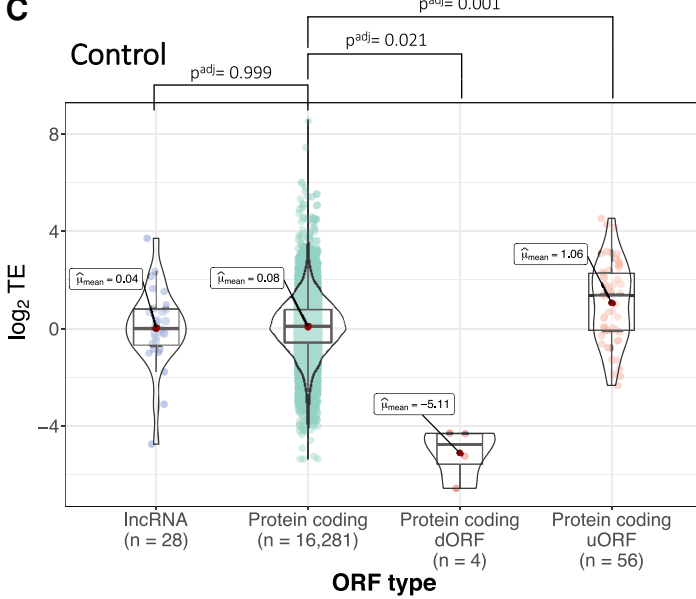

B

IncRNA-smORFs translated
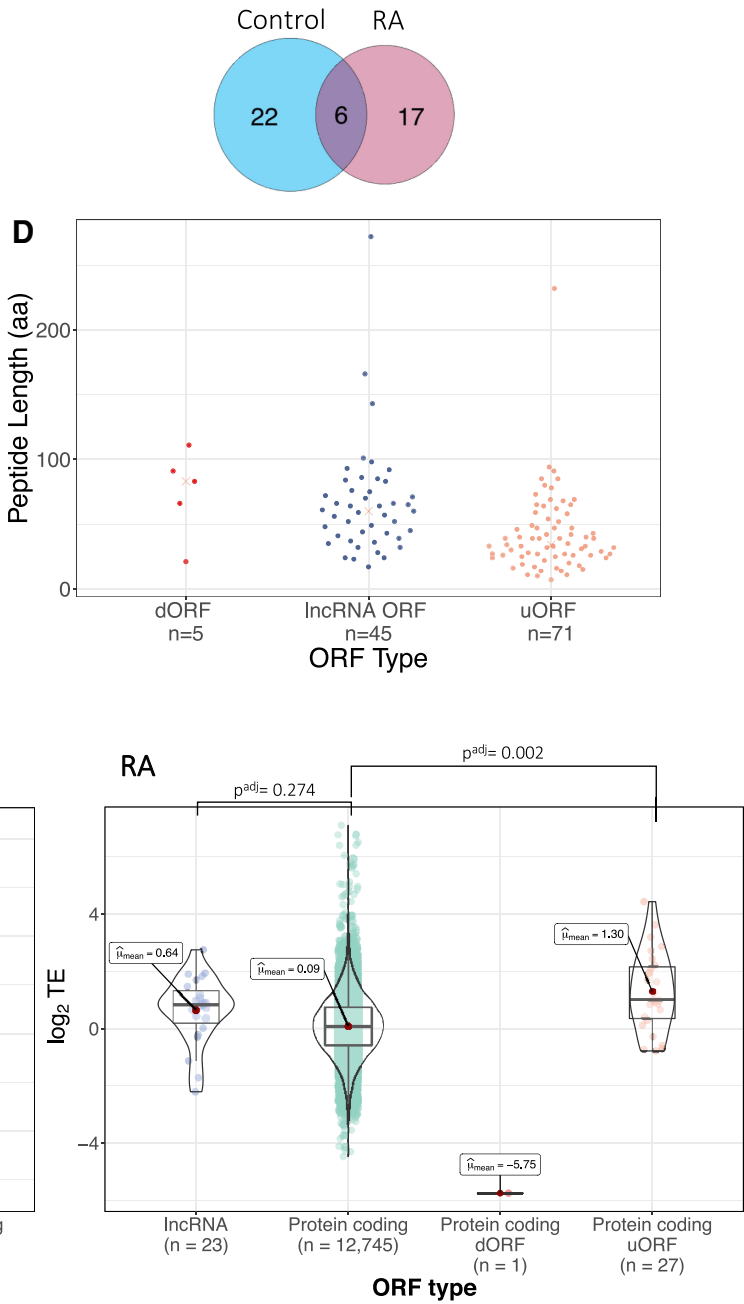

\section{E}
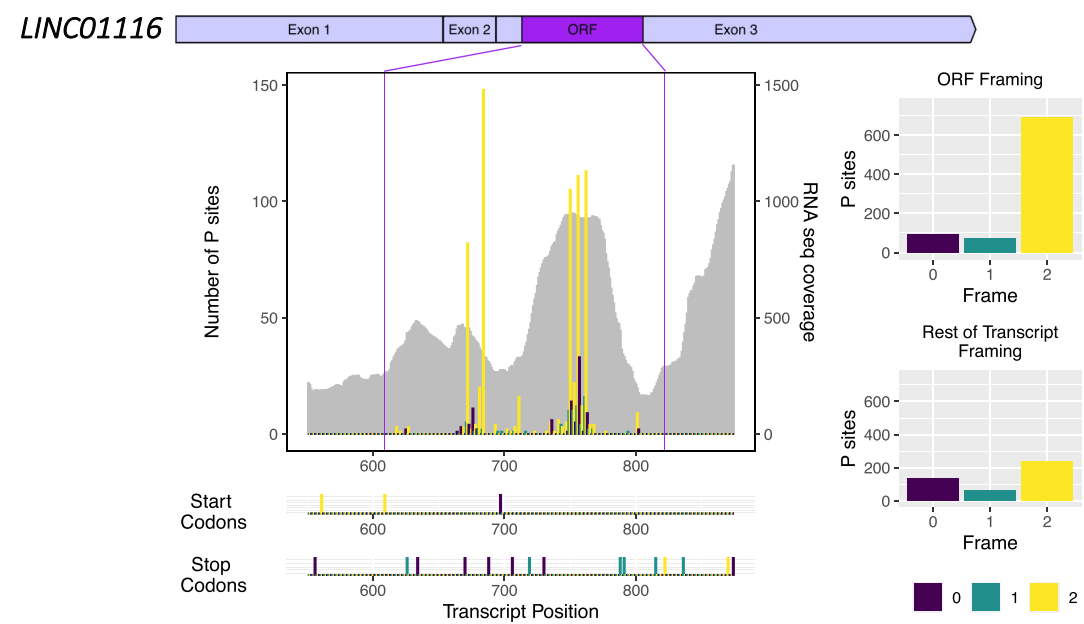

FIGURE 3. Translation of IncRNA-smORFs. (A) Workflow for identification of translated ORFs from Ribo-Seq and RNA-seq; see Materials and Methods for details. (B) Venn diagram of IncRNA-smORFs translated in Control and RA, with overlap. (C) Plots of translational efficiencies for protein-coding ORFs, IncRNA-smORFs, dORFS, and uORFs. (D) Length distribution of translated ORFs in IncRNAs, dORFs, and uORFs (in codons). (E) Poly-Ribo-Seq profile for LINC01116 in RA treatment. RNA-seq (Polysome) reads are gray and ribosome P sites are in purple, turquoise, and yellow according to frame. Purple lines mark beginning and end of translated smORF. All possible start and stop codons are indicated below. Framing within and outside translated smORF shown on left. 
TABLE 1. Translation of small ORFs

\begin{tabular}{lrrrr}
\hline Translated ORFs & Control & RA & Overlap & Total \\
\hline Protein-coding ORFs & 16,282 & 12,745 & 10,014 & 19,013 \\
uORFs & 56 & 27 & 12 & 71 \\
dORFs & 4 & 1 & 0 & 5 \\
IncRNA-smORFs & 28 & 23 & 6 & 45 \\
\hline
\end{tabular}

Number of ORFs detected as translated in Poly-Ribo-Seq.

Ribo-Seq from LINC00478 in both conditions (Fig. 4C). Transfection of LINC00478-smORF-FLAG into undifferentiated SH-SY5Y cells produced FLAG signal in both nuclear and cytoplasmic compartments (Fig. 4D). FLAG signal was also seen when LINC00478-smORF-FLAG transfected SHSY5Y cells were treated with RA (Supplemental Fig. 7A). This RA FLAG signal was only ever detected in the nucleus. Similar results were seen in HEK293 cells (Supplemental Fig. $7 \mathrm{~B})$, but because of the higher transfection efficiency in HEK293 compared with SH-SY5Y cells, we detected FLAG signal in more cells. Together this indicates that translation of LINC00478-smORF results in the synthesis of peptide, and the specific localization of this peptide is indicative of peptide function.

The second candidate IncRNA-smORF detected by our Poly-Ribo-Seq that we tagged was in LINC01116. Tagging of this LINC01116-smORF (Fig. 4E) generated a FLAG signal in the cytoplasm of SH-SY5Y cells, which is localized to neurites (Fig. 4F). A FLAG signal was also present in LINC01116 transfections in HEK293 cells (Supplemental Fig. 7C), but because of the higher transfection efficiency in HEK293 compared with SH-SY5Y cells, we detected a FLAG signal in more cells.

The LINC01116-smORF detected by Poly-Ribo-Seq is 71 codons in length, but inspection of the IncRNA sequence upstream of the smORF reveals a second potential ATG start codon (Fig. 4E; Supplemental Fig. 7D). Although there was little Ribo-Seq signal to support this $5^{\prime}$ start codon, it is possible that translation of LINC01116-smORF initiates there. The two potential start codons were assessed for similarity to the Kozak sequence consensus, using NetStart1.0 (Pedersen and Nielsen 1997); both exhibited scores $>0.5$, indicating that both are in good context and therefore either could be used to initiate translation $\left(\mathrm{AUG}_{1}=0.545, \mathrm{AUG}_{2}\right.$ $=0.645$ ). Given the scanning model of translation initiation it seems likely that the first AUG would be used. To determine if the $5^{\prime}$ start codon was used, it was mutated and the effect on production of a FLAG signal measured (Fig. 4E). No FLAG signal was present in transfections where the $5^{\prime}$ start codon was mutated ( $\Delta 1)$ (Fig. $4 \mathrm{G}$ ). This suggests that the first start codon is necessary for the translation of the LINC01116-smORF and the resulting peptide is 87 aa long. Although the FLAG signal is present in a low number of cells, no transfection controls and $\Delta 1$ indicate that the FLAG signal is dependent on translation of the LINC01116-smORF (Supplemental Fig. 7E). These results indicate that translation of LINC01116-smORF results in peptide synthesis and this 87 aa peptide exhibits a distribution suggestive of a function in neuronal differentiation.

\section{Translated IncRNA-smORFs exhibit sequence conservation}

Another indicator of coding potential and of peptide functionality is sequence conservation. Therefore, we assessed the extent to which the sequences of our novel IncRNAsmORFs are conserved. Given that IncRNAs in general are poorly conserved, we used closely related species to humans: the other four great apes ( $P$. abelii, $P$. paniscus, $P$. troglodytes, G. gorilla) as well as $N$. leucogenys (ape) and $M$. musculus. To ensure detection of sequence conservation for these short smORFs irrespective of annotation in other genomes, we used three complementary BLAST strategies using the transcript nt sequence, smORF nt sequence, and protein aa sequence.

Initial searches using the entire IncRNA transcript sequence (nt) and BLASTn (Altschul et al. 1990) returned results for $\sim 78 \%$ of translated IncRNAs (35/45), many of which had short alignment lengths of 30-100 nt. Although some of these results may represent conservation of the smORFs, many are due to small areas of sequence overlap along the rest of the IncRNA. LncRNAs rarely exhibit the same levels of conservation as mRNAs (Johnsson et al. 2014) but may contain short "modules" of higher sequence conservation, as described for XIST IncRNA (Brockdorff 2018). To take account of this, a second round of searches was performed on the initial search results, using the nt sequence of the smORF (BLASTn) (Altschul et al. 1990) followed by manual cross validation. This identified 14 IncRNA-smORFs as exhibiting nt sequence conservation in at least one of the apes or mouse (Fig. 5A). For the majority of these conserved IncRNA-smORFs, conservation is high across the smORF sequence and lower across the rest of the transcript. One such example is AL162386.2-smORF (ENST00000442428.1); it exhibits high sequence conservation when compared to gorilla (G. gorilla) and orangutan ( $P$. abelii), with $100 \%$ and $99 \%$ smORF nt sequence identity, respectively (Fig. 5B). When entire transcripts are aligned, this percentage sequence identity drops to $74 \%$ with gorilla (ENSGGOTO0000060708.1), and 65\% with orangutan (ENSPPYT00000022401.2), indicating the smORF is the most conserved part of these transcripts. Together this suggests that the AL162386.2-smORF, like canonical proteincoding CDSs is under greater selective pressure than its UTRs.

To further corroborate these results, a tBLASTn (Altschul et al. 1990) search of the IncRNA-smORF aa sequences was performed, which uses translated transcript databases in all six frames. This removes the noise of synonymous 
A

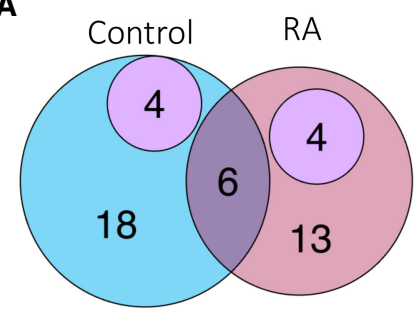

B

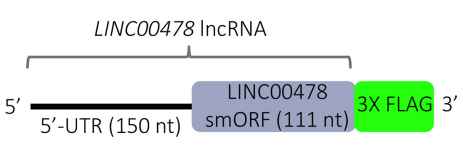

C

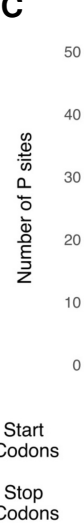

LINC00478 ORF, RA Rep 3

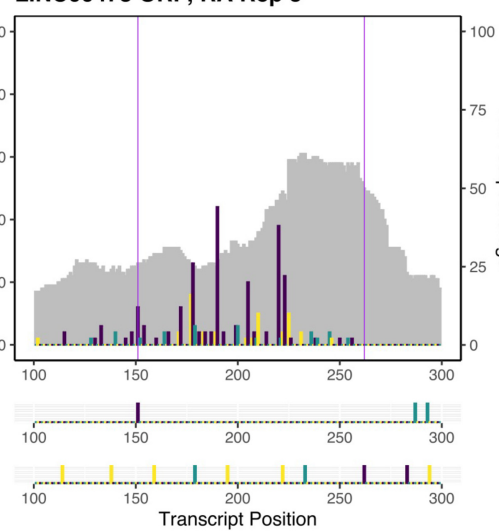

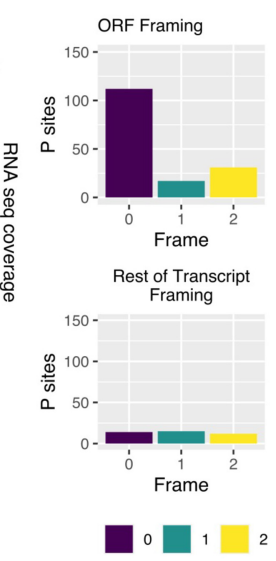

D

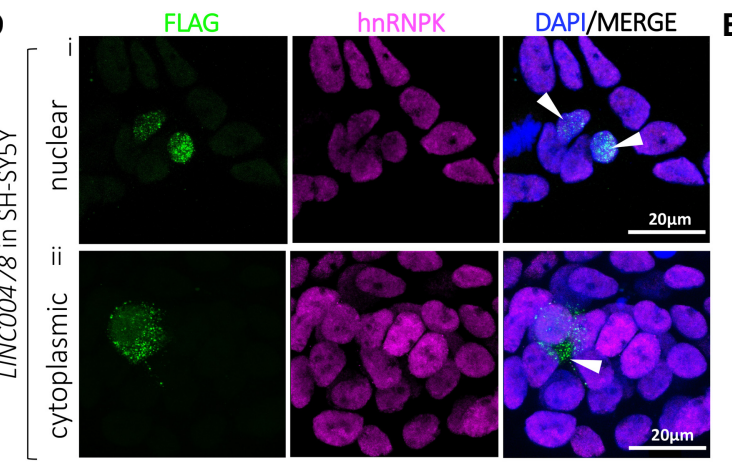

$E$

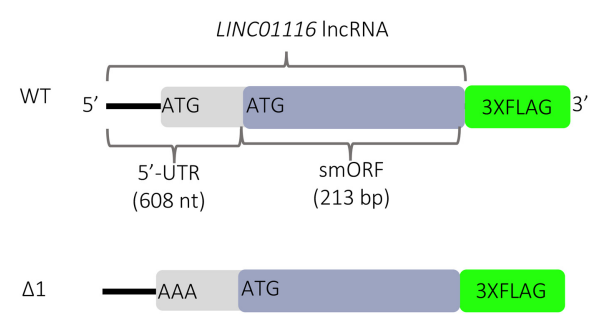

$\mathbf{F}$
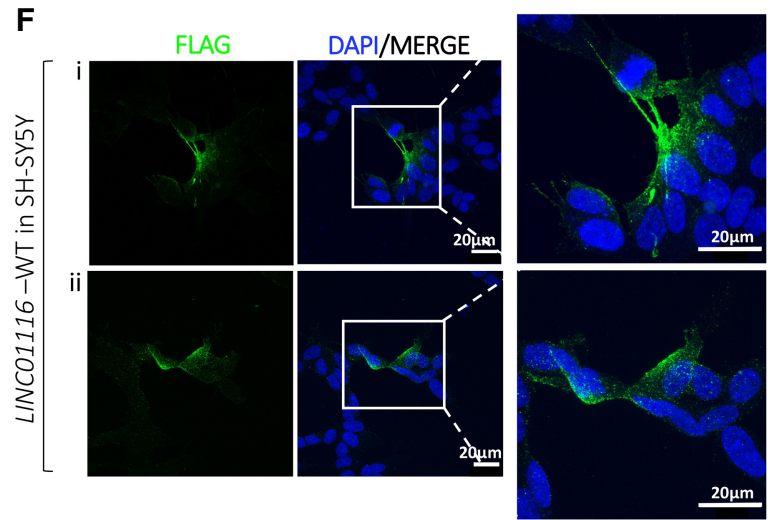

G

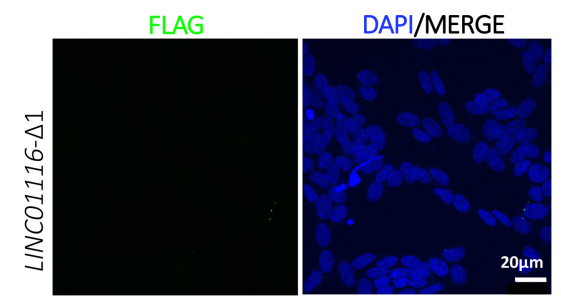

FIGURE 4. Peptide production from smORFs in IncRNAs. (A) Venn diagram showing overlap IncRNA-smORFs detected between our Poly-RiboSeq and publicly available mass spectrometry data from SH-SY5Y (purple). Control in blue, RA in pink. (B) Schematic of tagging construct for LINC00478; IncRNA sequence upstream of smORF and smORF, excluding its stop codon, cloned upstream of $3 \times$ FLAG, which is lacking its own start codon. FLAG signal is therefore dependent on smORF translation. (C) Poly-Ribo-Seq profile for LINC00478 in RA treatment. RNAseq (Polysome) reads are gray and ribosome $\mathrm{P}$ sites are in purple, turquoise, and yellow according to frame. Purple lines mark beginning and end of translated smORF. All possible start and stop codons are indicated below. Framing within and outside translated smORF shown on right. (D) Confocal images of FLAG-tagged LINC00478 peptide in SH-SY5Y cells (Control), showing (i) nuclear and (ii) cytoplasmic distribution, green is FLAG, magenta is hnRNPK (marking nuclei), and blue is DAPI (scale bar is $20 \mu \mathrm{m}$ ). (E) Schematic of tagging constructs for LINC01116 (WT and start codon mutant $\Delta 1$ ). (F) Confocal images of FLAG-tagged WT LINC01116 peptide showing cytoplasmic localization, near cell membrane and neuritic processes (magnification of insert is $3 \times$ ). (G) $\Delta 1$ start codon mutant, showing no FLAG signal, in SH-SY5Y cells; green is FLAG and blue is DAPI (scale bar is $20 \mu \mathrm{m}$ ).

substitutions, which can have a significant effect, particularly in smORFs (Ladoukakis et al. 2011). For the majority of smORFs, the same results were returned as the first BLASTn strategy, and evidence of conservation was found for a further three IncRNA-smORFs (ENST000004549 35.1_477_633, ENST00000557660.5_42_186, ENST000 00453910.5_151_262) that appear to have undergone some frameshift mutations (Fig. 5A). 


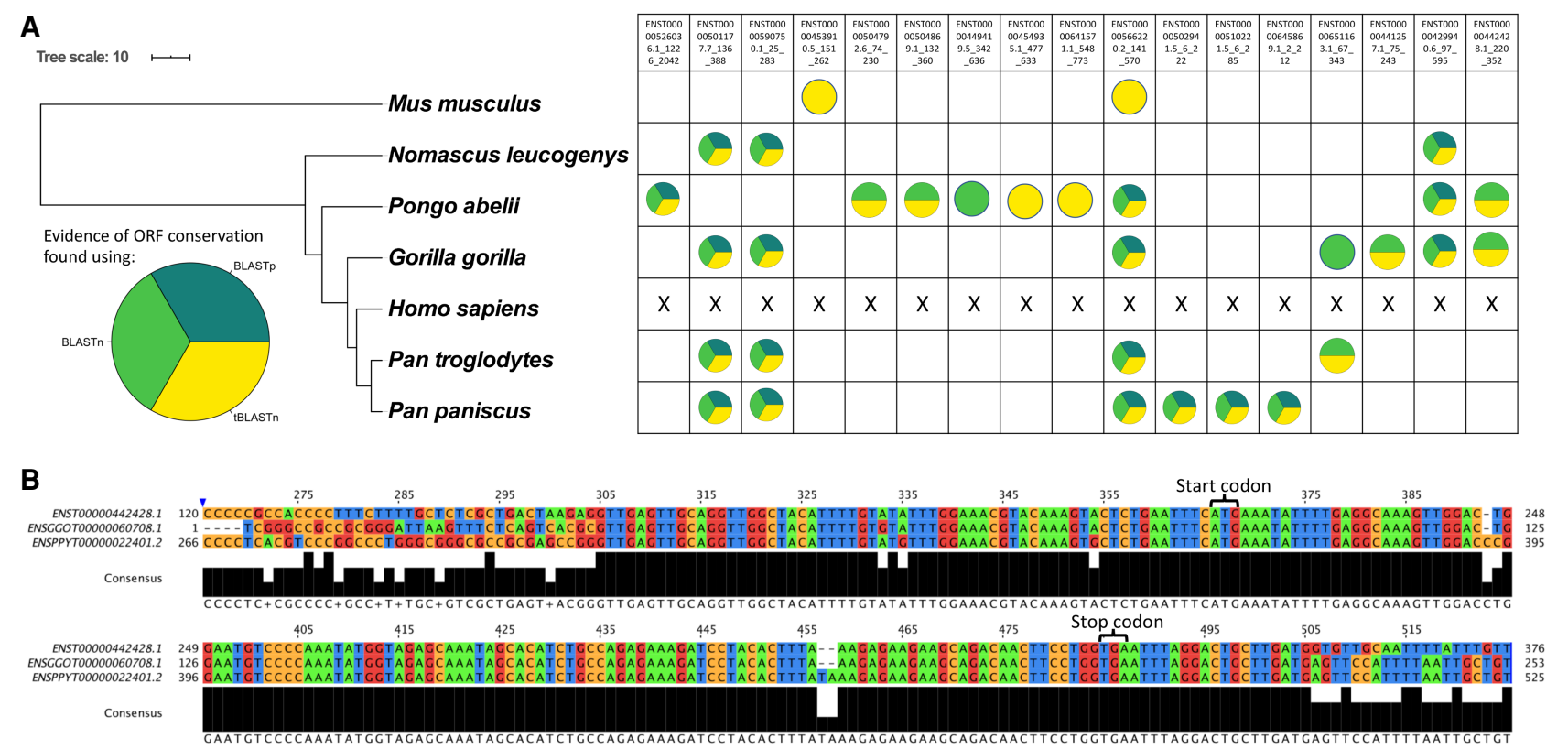

FIGURE 5. Translated IncRNA-smORFs exhibit sequence conservation in great apes. (A) Phylogram with IncRNA-smORFs for which evidence of sequence conservation was found represented as circles, colored according to how sequence conservation was identified. Phylogram built in iTOL (Letunic and Bork 2011) using data from TimeTree (Kumar et al. 2017), scale in 10 MYA. (B) Portion of human ENST00000442428 IncRNA nt alignment with gorilla and orangutan nt sequences, showing the smORF. Alignment built in ClustalOmega (Sievers et al. 2011) displayed in JalView (Waterhouse et al. 2009).

The third BLAST strategy at the aa sequence level, using BLASTp (Altschul et al. 1990), identified homologous peptide sequences for $18 \%(8 / 45)$ of the translated IncRNAsmORFs. This strategy only identifies peptide sequences previously annotated at protein-coding regions in the search species. However, all the returned proteins were unreviewed, uncharacterized proteins, with no evidence at protein, transcript, or homology levels in the Uniprot database (Bateman et al. 2019). This potentially suggests that automated annotation pipelines recognized the coding potential of these smORFs, unlike in the more curated human genome annotation. Overall, the combination of these three layers of sequence conservation analysis, as assessed by three BLAST strategies, revealed that 12 of our translated IncRNA-smORFs exhibit sequence conservation within Hominidae, three additional smORFs are also found in gibbons (Fig. 5B), with evidence for two translated smORFs detectable at the greater evolutionary distance of human to mouse, but not found in all apes (Fig. 5A). In total, we have discovered homologs for 17/45 of the IncRNA-smORFs in at least one of the searched species (Fig. 5A). Together this analysis indicates $38 \%$ of translated IncRNA-smORFs exhibit some level of sequence conservation, indicating that it is likely that they are translated in other species.

We took advantage of the identification of homologous smORFs in other species to determine if our translated smORFs exhibit signals of conserved protein-coding regions. Multispecies nt sequence alignments were made and phylogenetic codon analysis performed with PhyloCSF (Lin et al. 2011). Of the 17 IncRNA-smORFs with homologs in other species, 14 could be assessed, based on the organisms that the homologs were identified in. Four smORFs exhibited positive PhyloCSF scores, indicating that they are likely to represent a conserved coding region, while the other 10 had negative scores (Supplemental Table 2). The four positive scoring IncRNA-smORFs are in AC020928.2, ENTPD1-AS1, LINC00839, and THAP9-AS1. This analysis provides further support that these four IncRNA-smORFs likely encode peptides.

\section{Translated IncRNAs are associated with neuronal functions and diseases}

To probe the potential biological function, expression, and association with human disease, we profiled the 45 translated IncRNAs using published data. Dynamic expression of IncRNAs can indicate tissues and developmental time points when IncRNAs may function. Therefore, we first assessed whether our translated IncRNA genes exhibited developmentally dynamic expression. In IncExpDB (Sarropoulos et al. 2019), genes that show large changes in expression at different time points during development of a specific organ are annotated as "dynamic." Thirty-nine percent of our translated IncRNA genes exhibit "dynamic" expression compared with $19 \%$ of the total population of IncRNA genes present in IncExpDB (Fig. 6A). This indicates 
that translated IncRNA genes are more likely to have developmentally regulated expression than IncRNAs in general, suggesting biological roles for these IncRNAs.

We found that $62 \%$ of these dynamic translated IncRNAs are regulated in the brain, compared with $20 \%$ of all dynamic IncRNAs (Fig. 6B), therefore our translated IncRNAs may function in the brain during development. When we consider our own RNA-seq of Control and RA treated SH-SY5Y cells, $22 \%$ of the translated IncRNAs exhibit differential expression in the cytoplasm, 20\% up-regulated and $2 \%$ down-regulated upon differentiation (Fig. 6C). This potentially indicates that the biological role of these translated IncRNAs may be of broader neuronal importance than just in this differentiation model.

By examining data from the FANTOM6 project, we were able to probe potential cellular functions for three of our translated IncRNAs. siRNA knockdowns of LINC01116, FGD5-AS1, and TUG1 were performed in human dermal fibroblasts followed by RNA-seq to understand global effects of depleting these IncRNAs. GO term analysis of these published data showed that genes associated with neuronal function were enriched for all three of our translated IncRNAs (Fig. 6D). This is particularly striking given knockdown was performed in a nonneuronal cell type and suggests all three IncRNAs possess neuronal functions.

To investigate potential roles for our translated IncRNAs in human disease, we examined published association studies specifically for neurological diseases and disorders (Chen et al. 2013; Li et al. 2016; Rappaport et al. 2017; Bao et al. 2019). This revealed $68 \%$ of the translated IncRNAs have an association with cancers of the central nervous system (CNS) (Fig. 6E). This is consistent with our discovery of their translation in a neuroblastoma cell line (SH-SY5Y). In addition, $21 \%$ of the translated IncRNAs are associated with neurodegenerative diseases and $18 \%$ with neurodevelopmental disorders (Fig. 6E). Overall examination of published data on our translated IncRNA indicates that they likely have neuronal functions, potentially during neuronal development, and may contribute to neuronal diseases.

\section{LINC01116 contributes to neuronal differentiation}

To dissect the potential role of the translated IncRNAs during neuronal differentiation, we performed siRNA knockdown in SH-SY5Y cells. We selected the candidate IncRNA LINC01116 because we discovered it is induced during differentiation and translated to produce a neurite localized peptide. We performed LINC01116 siRNA knockdown in both undifferentiated and differentiated SH-SY5Y cells, achieving an 89\%-94\% reduction in LINC01116 levels (Supplemental Fig. 8A). LINC01116 knockdown had a limited effect on cell viability (Supplemental Fig. 8B). The extent of differentiation was then assessed by Tuj1 immunofluoresence, which revealed that LINC01116 knockdown resulted in a significant reduction of neurite length in RA treated SH-SY5Y cells (Fig. 7A, zoom in Fig. 7B), compared to scrambled siRNA treated SH-SY5Y (Fig. 7C). However, there was no effect of the knockdown in undifferentiated cells (Fig. 7C). This suggests that LINC01116 is involved in the regulation of neuritic processes formation during neuronal differentiation. To examine potential effects of LINC01116 knockdown further on differentiation, we assessed the expression levels of the noradrenergic marker MOXD1, which is important in neural crest development. LINC01116 siRNA knockdown, upon differentiation, resulted in a reduction of MOXD1 expression levels, further indicating a role of LINC01116 in neuronal differentiation (Fig. 7D). However, LINC01116 knockdown had no effect on proliferation, as measured by percentage of Ki67+ cells (Supplemental Fig. 8C,D) or cell cycle, as measured by E2F1 mRNA RT-qPCR (Supplemental Fig. 8E). LINC01116 likely functions early in the differentiation pathway since its levels are significantly up-regulated within the first $24 \mathrm{~h}$ of RA-induced differentiation (Supplemental Fig. 8F). Expression of LINC01116 then declines rapidly by day 8 (Supplemental Fig. 8G). Together these results suggest that LINC01116 functions during early differentiation, contributing to neurite process formation.

\section{DISCUSSION}

In this work, we have dissected the relationship of IncRNAs with the translation machinery during human neuronal differentiation using RA treated SH-SY5Y cells as a model. We discovered that $\sim 800-900$ IncRNA genes are expressed and exported to the cytoplasm. A total of $85 \%-90 \%$ of these cytoplasmic IncRNAs are associated with polysome complexes, suggesting that they are either being translated or regulating the translation of the mRNAs with which they interact. Moreover, the association of IncRNAs with polysomes is dynamic during differentiation, as shown by the differential polysome enrichment of IncRNAs in Control and RA treated cells. These results reveal that many IncRNAs are present in the cytoplasm, enriched there, and associated with translation complexes.

We characterized LINC02143 in more detail, which was found to associate with polysomes. It is an intergenic IncRNA with no known function, which is induced upon differentiation. It is detected in $80 \mathrm{~S}$ and small polysome fractions, indicating it interacts with the translation machinery, but it is not detected as translated. A number of antisense polysome-associated IncRNAs appear to be up-regulated upon differentiation. Among them is DLGAP1-AS1, which is antisense to Disks large-associated protein 1 (DLGAP1), a protein-coding gene involved in chemical synaptic transmission. DLGAP1-AS1 interacts with actively translating polysomes both in Control and upon differentiation, but it is not translated. The IncRNAs depleted from the 
A

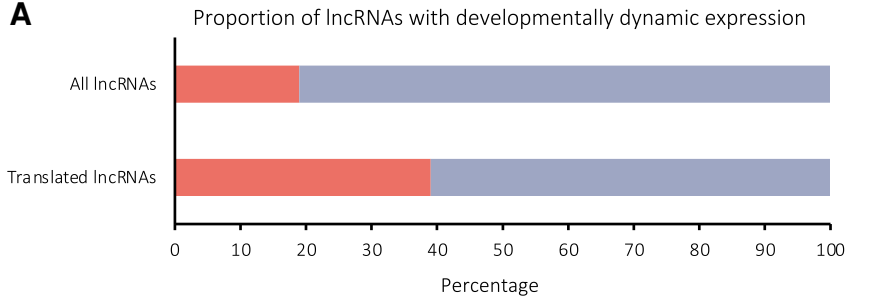

B

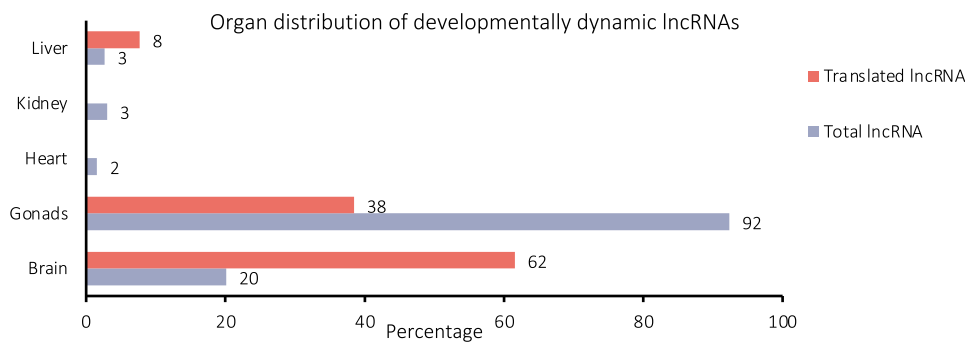

C

Regulation of translated IncRNAs during differentiation

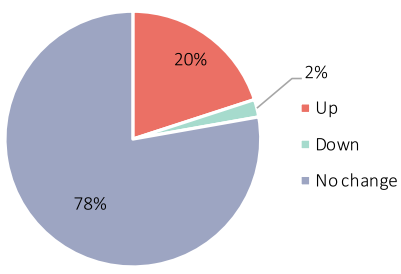

E Percentage of translated IncRNAs associated with neurological diseases and disorders

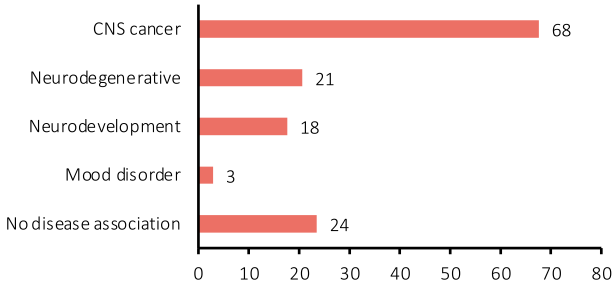

D

\begin{tabular}{|c|c|}
\hline InCRNA & Downregulated pathways and Normalised enrichment score \\
\hline LINC01116 & $\begin{array}{l}\text { GO_MYELIN_SHEATH }-1.488 \\
\text { GO_NEURON_MIGRATION - } 1.625 \\
\text { GO_LARGE_RIBOSOMAL_SUBUNIT }-2.437 \\
\text { GO_SMALL_RIBOSOMAL_SUBUNIT }-2.436 \\
\text { GO_POSITIVE_REGULATION_OF_CANONICAL_WNT_SIGNALING_PATHWAY }-1.889\end{array}$ \\
\hline FGD5-AS1 & $\begin{array}{l}\text { GO_NEURON_PROJECTION_GUIDANCE - } 2.086 \\
\text { GO_NEURON_PROJECTION_MEMBRANE }-2.069 \\
\text { GO_NEURON_RECOGNITION }-2.066 \\
\text { GO_MAIN_AXON - } 1.99 \\
\text { GO_SYNAPTIC_SIGNALING }-1.981 \\
\text { GO_NEURON_PROJECTION_MORPHOGENESIS }-1.958\end{array}$ \\
\hline TUG1 & $\begin{array}{l}\text { GO_SYNAPTIC_MEMBRANE-1.885 } \\
\text { GO_SMAD_BINDING - } 1.885 \\
\text { GO_NEGATIVE_REGULATION_OF_WNT_SIGNALING_PATHWAY - } 1.708 \\
\text { GO_NEURON_PROJECTION_MORPHOGENESIS-1.836 } \\
\text { GO_CELL_MORPHOGENESIS_INVOLVED_IN_NEURON_DIFFERENTIATION-1.820 } \\
\text { GO_CANONICAL_WNT_SIGNALING_PATHWAY-1.800 }\end{array}$ \\
\hline
\end{tabular}

FIGURE 6. Potential biological importance of translated IncRNAs in neural development, differentiation, and disease. (A) Percentage of translated IncRNA genes and all IncRNA genes, which exhibit dynamic expression during human development according to IncExpDB (Sarropoulos et al. 2019). (B) Percentage of the dynamically expressed, translated IncRNAs, which show dynamic expression in each organ, for total and translated IncRNA populations, according to LncExpDB (Sarropoulos et al. 2019). (C) Proportion of translated IncRNAs, which exhibit differential expression during differentiation of $\mathrm{SH}-\mathrm{SY} 5 Y$ cells. (D) GO terms associated with changes in RNA-seq levels upon siRNA knockdown of three of the translated IncRNAs (LINC01116, FGD5-AS1, and TUG1) performed by FANTOM6 in human dermal fibroblasts. (E) Percentage of translated IncRNA genes found to be associated with neuronal diseases and disorders according to IncRNADisease, Differential Expression Atlas, Cancer RNA-Seq Nexus, Malacards.

polysomes have fewer antisense IncRNAs relative to other populations, suggesting that antisense IncRNAs are preferentially localized to polysomes. These polysome-associated antisense IncRNAs could potentially regulate the translation of their "sense" mRNA through base-pairing, as is the case with BACE1-AS (Faghihi et al. 2010) and UCHL1-AS (Carrieri et al. 2012).

Ribosome profiling of the actively translating polysomes allowed us to distinguish between the IncRNAs that simply associate with the polysome complexes and those that are being actively translated. We identified 45 translated IncRNA-smORFs, 43 of which are novel ORFs. These translated IncRNA-smORFs exhibit high levels of triplet periodicity, and their translational efficiencies are similar to protein-coding genes. We can therefore be confident that these are real translation events leading to the production of substantial peptide levels rather than background, spurious events (Guttman et al. 2013; Bazzini et al. 2014; Ruiz-Orera and Alba 2019; Patraquim et al. 2020). The size distribution of our novel translated ORFs indicates that the majority are indeed smORFs (<100aa). The general pattern we identified is that dORFs $>$ IncRNA-smORF $>$ uORFs in size. This is consistent with previous studies where a wide range of peptide lengths were discovered (Aspden et al. 2014; Chong et al. 2020). Amino acid composition of these translated smORFs supports the fact they are translated into peptides. However, it does not suggest they are enriched for transmembrane $\alpha$-helices, in contrast to the smORFs characterized in D. melanogaster (Aspden et al. 2014).

Overall, we have independent evidence for peptide synthesis for 12/45 IncRNA-smORFs. Eight of these are from published mass spectrometry data from SH-SY5Y cells (Brenig et al. 2020). In general, we find our IncRNA-smORFs translated in the same treatment (undifferentiated or differentiated) as these mass spectrometry data sets detect the smORF peptides (7/8). An 18\% mass spectrometry detection level may seem low but given the limitations of detecting small peptides by mass spectrometry, this represents a substantial level of validation. Two translation events were validated by FLAG tagging transfection assay: LINC01116 and 
A
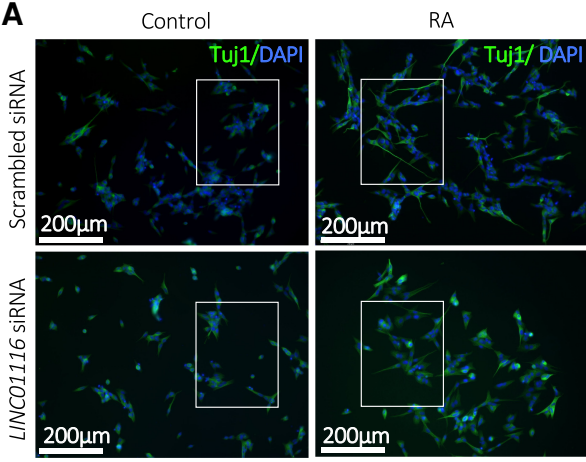

C

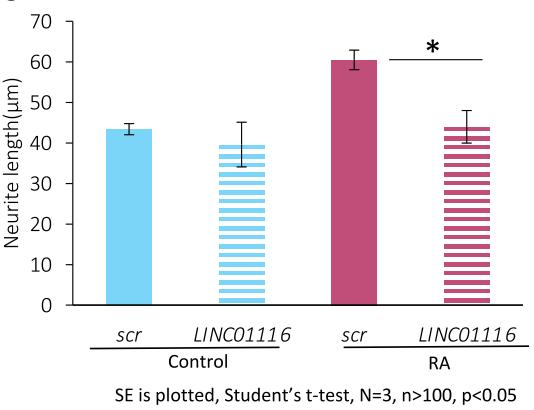

B

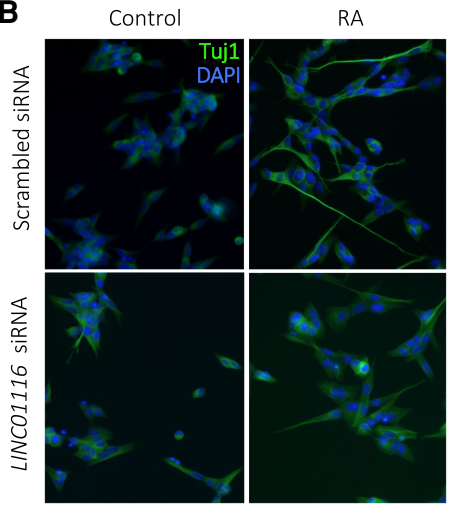

D

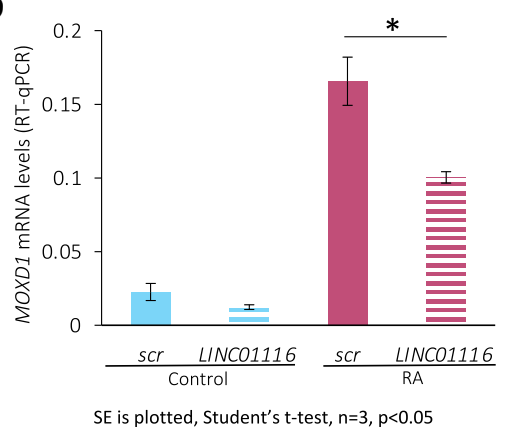

FIGURE 7. LINC01116 contributes to neuronal differentiation but does not affect cell cycle progression. (A) Representative immunofluorescence images of Control and RA SH-SY5Y cells, transfected with siRNA targeting LINC01116 and scrambled control, after staining for Tuj ( $\beta$ III-tubulin) at day 3 post-differentiation (scale bar $=200 \mu \mathrm{m}$ ). White windows magnified in B. (C) Quantification of neurite length in Control and RA treated cells upon knockdown shows a significant reduction of neurite length in the differentiated cells upon LINC01116 knockdown ( $N=3$ biological replicates, $n>100$ measurements, Student's t-test $P<0.05$ ). (D) RT-qPCR of differentiation marker MOXD1 in Control and RA treated cells, transfected with siRNA targeting LINC01116 and scrambled control, shows significant reduction of MOXD1 expression in differentiated cells with reduced LINC01116 levels at day 3 post-differentiation ( $n=3$ biological replicates, Student's $t$-test $P<0.05)$.

LINC00478 IncRNA-smORFs. The production of $2 / 45$ IncRNA-smORF peptides is corroborated by previous studies in nonneuronal cells. HAND2-AS1 (translated in Control and RA) is translated in human and rodent heart and encodes for an integral membrane component of the endoplasmic reticulum (van Heesch et al. 2019). CRNDE, which is only translated upon differentiation, encodes for a previously characterized nuclear peptide (CRNDEP) (Szafron et al. 2015). The translation of these smORFs in multiple cell types provides substantial support for the production of peptides and their potential function.

We also discovered that $24 \%$ of the IncRNA-smORFs we find translated show sequence conservation across Hominidae. This suggests that the other great apes have the potential to translate very similar peptides. This provides additional evidence to indicate that these translation events are not translational noise. Of course, it will be interesting to uncover the function of these small peptides in the future. Four of the conserved IncRNA-smORFs are under purifying selection and therefore likely to encode peptides.

LINC01116-smORF DNA sequence is on the opposite strand to a SINE element, suggesting that this IncRNA and smORF have likely evolved from a SINE transposable element (TE). This is consistent with previous observations that $39 \%$ of IncRNA sequences are derived from TEs (Carlevaro-Fita et al. 2016) and that LINC01116 is human specific, that is, not found in other apes. Many other small ORFs have also been found to originate from Alu elements; for example, 287 human uORFs (Shen et al. 2011). Together this suggests that LINC01116-smORF has recently evolved from a TE.

We found that $22 \%$ of the translated IncRNAs are differentially expressed during neuronal differentiation of $\mathrm{SH}$ SY5Y. Analysis of publicly available data sets revealed that our translated IncRNAs show regulated expression during human development, specifically in the brain, more so than IncRNAs in general. Many of these translated IncRNAs also exhibit associations with neuronal diseases. The FANTOM6 project data suggests that LINC01116, FGD5-AS1, and TUG1 have cellular roles in neuronal function (Ramilowski et al. 2020). Together this indicates that these translated IncRNAs play roles in neuronal development and differentiation and likely contribute to neurological diseases.

Here we have discovered that LINC01116 produces an 87aa peptide that exhibits cytoplasmic localization, and specifically is detected near the cell membrane and 
in neuritic processes. The up-regulation of LINC01116 expression upon differentiation, coupled with the localization of its peptide, led us to further investigate its potential role in differentiation. Knockdown of LINC01116 upon differentiation appears to impede neurite outgrowth and results in the reduction of the mRNA levels of the noradrenergic marker MOXD1. Our data suggest that LINC01116 is involved in the regulation of neuronal differentiation, consistent with the fact that it is moderately expressed in the developing human forebrain and highly expressed in the developing human midbrain and spinal cord (Lindsay et al. 2016). The effects of siRNA in human dermal fibroblasts also supports a role for LINC01116 in neuronal migration (Ramilowski et al. 2020). LINC01116 has previously been found to be involved in two other cancer models: in the progression of glioblastoma (Brodie et al. 2017); and it is up-regulated in gefitinib resistant non-small cell lung cancer cells (Wang et al. 2020). siRNA knockdown of LINC01116 in both these cell types results in decreased expression of stem-cell markers (NANOG, SOX2 and OCT4) and reduced cell proliferation. This suggests LINC01116 promotes cell proliferation in these systems, indicating that the downstream effects of LINC01116 may vary according to cell type. However, knockdown of LINC01116 also inhibited migration of glioma stem cells (Brodie et al. 2017), while overexpression of LINC01116 promoted invasion and migration of gastric cancer cells (Su et al. 2019). This suggests a potential role of LINC01116 in the formation of cell membrane protrusions, which is consistent with the role we have discovered for LINC01116 in neurite development. It is yet to be determined if this function of LINC01116 during neuronal differentiation is performed at the IncRNA or peptide level.

To conclude, our findings indicate that many IncRNAs are localized in the cytoplasm and they likely play functional roles as indicated by their regulation during differentiation and polysome association. Given the large number of IncRNAs we found to be associated with polysomes in the cytoplasm, it is likely that future work will assign the functions of many more IncRNAs to translational regulation. We have identified 43 novel translation events, many of which are regulated during differentiation. The IncRNAsmORFs we discover here represent a general population whose products have not yet been characterized. As demonstrated for LINC01116, IncRNAs and the small peptides encoded therein have the potential to contribute to important cellular functions, development, and disease.

\section{MATERIALS AND METHODS}

\section{Cell culture}

Human neuroblastoma SH-SY5Y cells, were cultured in Dulbecco's Modified Eagle Medium (DMEM 4.5g/L Glucose with L-Glutamine) supplemented with $1 \%(\mathrm{v} / \mathrm{v})$ Penicillin/Streptomycin and
$10 \%$ Fetal Bovine Serum (FBS) at $37^{\circ} \mathrm{C}, 5 \% \mathrm{CO}_{2}$. Neural induction commenced at passage 4 and was performed as described previously (Korecka et al. 2013; Forster et al. 2016) with minor alterations. All trans Retinoic Acid (RA, Sigma) was added to cells $24 \mathrm{~h}$ after plating, at a final concentration of 30 $\mu \mathrm{M}$ for $3 \mathrm{~d}$.

\section{Immunocytochemistry}

Cells were seeded on Poly-D-Lysine/mouse laminin coated $12 \mathrm{~mm}$ round coverslips (Corning BioCoat Cellware) and fixed with $4 \%$ paraformaldehyde (PFA) (Affymetrix) for $20 \mathrm{~min}$ at room temperature (RT). A permeabilization step $(0.1 \%$ Triton- $X$ for $10 \mathrm{~min}$ at RT) was performed prior to blocking, followed blocking at RT in blocking buffer (3\% BSA, $1 \times$ PBS or $5 \%$ NGS, $1 \times$ PBS and $0.1 \%$ Triton-X) for $30 \mathrm{~min}$. Primary antibodies (Supplemental Methods) were applied in 3\% BSA $1 \times$ PBS or $0.5 \%$ NGS, $1 \times$ PBS, $0.1 \%$ Triton-X and incubated at RT for $2 \mathrm{~h}$ or at $4^{\circ} \mathrm{C}$ overnight. Cells were washed and labeled with Alexa 488, Alexa 555 , or Alexa 633 at 1:500 dilution for $2 \mathrm{~h}$ at RT in 0.5\% NGS, $1 \times$ PBS, $0.1 \%$ Triton-X. Cells were mounted in VECTASHIELD mounting medium, analyzed using LSM 700 confocal microscope (Zeiss) ImageJ.

\section{CDNA synthesis and quantitative real time PCR (RT-qPCR)}

Equal amounts of RNA (whole cell, nuclear, and cytoplasmic lysates) or equal volumes (polysome fractions) were subject to cDNA synthesis, using qScript (Quantabio) according to manufacturer's instructions. QPCR was performed using the CFX Connect Thermal Cycler and quantification using SYBR Green fluorescent dye (PowerUp SYBR Green Master Mix, Thermo Fisher Scientific). Primers were designed to anneal to exonexon junctions, where possible, or to common exons between alternative transcripts (Supplemental Methods). Target mRNA and IncRNA levels were assessed by absolute quantification by the means of standard curve or relative quantification, using the $\Delta \Delta \mathrm{Cq}$ method.

\section{Polysome profiling}

RA was added to SH-SY5Y cells 3 d prior to harvesting. Cells were treated with cycloheximide (Sigma) at $100 \mu \mathrm{g} / \mathrm{mL}$ for $3 \mathrm{~min}$ at $37^{\circ} \mathrm{C}$, washed ( $1 \times$ PBS, $100 \mu \mathrm{g} / \mathrm{mL}$ cycloheximide) and trypsinized for $5 \mathrm{~min}$ at $37^{\circ} \mathrm{C}$. Subsequently, cells were pelleted, washed $(1 \times$ PBS, $100 \mu \mathrm{g} / \mathrm{mL}$ cycloheximide), and resuspended in ice cold lysis buffer (Supplemental Methods); 50 mM Tris-HCl pH 8, 150 mM $\mathrm{NaCl}, 10 \mathrm{mM} \mathrm{MgCl}$, $1 \mathrm{mM}$ DTT, 1\% IGEPAL, $100 \mu \mathrm{g} / \mathrm{mL}$ cycloheximide, Turbo DNase $24 \mathrm{U} / \mu \mathrm{L}$ (Invitrogen), RNasin Plus RNase Inhibitor 90U (Promega), cOmplete Protease Inhibitor (Roche), for $45 \mathrm{~min}$. Cells were then subjected to centrifugation at $17,000 \mathrm{~g}$ for $5 \mathrm{~min}$, to pellet nuclei. Cytoplasmic lysates were loaded onto $18 \%-60 \%$ sucrose gradients $\left(\sim 70 \times 10^{6}\right.$ cells per gradient) at $4^{\circ} \mathrm{C}$ and subjected to ultracentrifugation (121,355 $\times \mathrm{g}_{\text {avg }}$ $3.5 \mathrm{~h}, 4^{\circ} \mathrm{C}$ ) in SW-40 rotor. Gradients were fractionated using Gradient Station (Biocomp) and absorbance at $254 \mathrm{~nm}$ was monitored using a Bio-Rad detector. 


\section{Poly-Ribo-Seq}

Approximately $20 \%$ of cytoplasmic lysate was kept for poly(A) selection (total RNA control) and $~ 80 \%$ was loaded onto $18 \%-60 \%$ sucrose gradients $\left(\sim 70 \times 10^{6}\right.$ cells per gradient) at $4^{\circ} \mathrm{C}$ and subjected to ultracentrifugation $\left(121,355 \times \mathrm{g}_{\text {avg }} 3.5 \mathrm{~h}, 4^{\circ} \mathrm{C}\right)$ in $\mathrm{SW}$ 40 rotor. Polysome fractions were pooled from control and from differentiated cells. Approximately $25 \%$ polysomes were kept for $\operatorname{poly}(\mathrm{A})$ selection (polysome-associated RNA). The remaining $75 \%$ was diluted in $100 \mathrm{mM}$ Tris $-\mathrm{HCl} \mathrm{pH} 8,30 \mathrm{mM} \mathrm{NaCl}$, $10 \mathrm{mM} \mathrm{MgCl}$. RNasel (EN601, $10 \mathrm{U} / \mu \mathrm{L} 0.7-1 \mathrm{U} / \mathrm{million}$ cells) was subsequently added and incubated overnight at $4^{\circ} \mathrm{C}$. RNasel was deactivated using SUPERase inhibitor (200 U/gradient) for $5 \mathrm{~min}$ at $4^{\circ} \mathrm{C}$. Samples were concentrated using $30 \mathrm{kDa}$ molecular weight cutoff columns (Merck) and loaded on sucrose cushion (1 $\mathrm{M}$ sucrose, $50 \mathrm{mM}$ Tris- $\mathrm{HCl} \mathrm{pH} 8,150 \mathrm{mM} \mathrm{NaCl}$, $10 \mathrm{mM} \mathrm{MgCl}_{2}, 40 \mathrm{U}$ RNase Inhibitor) and subjected to ultracentrifugation at $204,428 \times \mathrm{gavg}_{\text {at }} 4^{\circ} \mathrm{C}$ for $4 \mathrm{~h}$ (TLA110). Pellets were resuspended in TRIzol (Ambion, Life Technologies) and processed for RNA purification.

RNA purification from cytoplasmic lysates and RNasel footprinted samples was performed by TRIzol RNA extraction, following manufacturer's instructions. RNA purification from polysome fractions was performed by isopropanol precipitation, followed by TURBO DNase treatment (Thermo Fisher) (according to manufacturer's instructions), acidic phenol/chloroform RNA purification and ethanol precipitation at $-80^{\circ} \mathrm{C}$ overnight. RNA concentration was determined by Nano-drop 2000 software. Two rounds of poly $(A)$ selection from total cytoplasmic lysate and polysome fractions were performed using oligo (dT) Dynabeads (Invitrogen) according to manufacturer's instructions. Poly(A) RNA was fragmented by alkaline hydrolysis. A total of 28$34 \mathrm{nt}$ ribosome footprints and 50-80 nt mRNA fragments were gel purified in $10 \%(\mathrm{w} / \mathrm{v})$ polyacrylamide-TBE-Urea gel at $300 \mathrm{~V}$ for 3.5 $h$ in $1 \times$ TBE. Ribosome footprints were subjected to rRNA depletion (Illumina RiboZero rRNA Removal Kit).

5' stranded libraries were constructed using NEB Next Multiplex Small RNA Library Prep. The resulting cDNA was PCR amplified and gel purified prior to sequencing. Libraries were subjected to 75 bp single end RNA-seq using NextSeq500 Illumina sequencer, High Output Kit v2.5 (75 Cycles) (Next Generation Sequencing Facility, Faculty of Medicine, University of Leeds).

\section{RNA-seq data analysis}

RNA-seq reads were trimmed with Cutadapt (v.19.1) (Martin 2011) and filtered with fastq_quality_filter (v.0.0.13) (Hannon $2010)$ to filter out the reads of low quality $(90 \%$ of the reads to have a phred score above 20). Filtered reads were mapped (Liao et al. 2013) to the human genome reference (the IncRNA GENCODE Release 19 [Frankish et al. 2019] and annotation added to mRNA annotation from the UCSC [Haeussler et al. 2019] human genome assembly [hg19] from iGenomes) with Rsubread (v.1.22.0) (Liao et al. 2013), and uniquely mapped reads were reported. Bam file sorting and indexing was performed with SAMtools (v.1.3.1) (Li et al. 2009). Subsequently summarized read counts for all genes were calculated using featureCounts (Liao et al. 2014). For normalization, RPKM values were calculat- ed. Differential expression analysis was conducted with DESeq2 (v.1.12.0) (Love et al. 2014) based on the two cutoffs $P^{\text {adj }}<0.05$ and the absolute value of $\log _{2}$ FoldChange $>1$. Gene ontology analysis was performed with GOrilla (Gene Ontology enRIchment anaLysis and visuaLizAtion tool) (Eden et al. 2009).

\section{Ribo-Seq analysis}

Quality reports of polysome-associated RNA-seq and Ribo-Seq data were made using Fastqc (v.0.11.9) (Andrews 2010). Adaptor sequences were trimmed using Cutadapt (v.210) (Martin 2011) with a minimum read length of $25 \mathrm{bp}$, and untrimmed outputs retained for RNA-seq reads. Low-quality reads (score $<20$ for $10 \%$ or more of reads) were then discarded using FASTQ Quality Filter, FASTX-Toolkit (v.0.0.14) (Gordon 2010). Human rRNA sequences were retrieved from RiboGalaxy (Michel et al. 2016) and high confidence hg38 tRNA sequences from GtRNAdb Release 17 (Chan and Lowe 2016). One base was removed from the $3^{\prime}$ ends of reads to improve alignment quality; reads originating from rRNA and tRNA were aligned and removed using Bowtie2 (v.2.4.1) (Langmead and Salzberg 2012).

The splice aware aligner STAR (v2.7.5c) (Dobin et al. 2012) was used to map remaining reads to the human reference genome (GRCh38.p12), GENCODE release 30 (Frankish et al. 2019). The STAR (v2.7.5c) (Dobin et al. 2012) genome index was built with a sjdbOverhang of 73. SAMtools (v.1.10) (Li et al. 2009) was used to create sorted, indexed bam files of the resulting alignments.

Metaplots of aligned Ribo-Seq data were generated using metaplots.bash script from Ribotaper (v1.3) (Calviello et al. 2016) pipeline. These show the distance between the $5^{\prime}$ ends of Ribo-Seq and annotated start and stop codons from CCDS ORFs, allowing the locations of P-sites to be inferred. Read lengths exhibiting the best triplet periodicity were selected for each replicate, along with appropriate offsets (Supplemental Fig. 5; Supplemental Table 1).

Actively translated smORFs were then identified using Ribotaper (v1.3) (Calviello et al. 2016). Initially, this requires an exon to contain more than five P-sites in order to pass to quality control steps. Identified ORFs were then required to have a 3-nt periodic pattern of Ribo-Seq reads, with $50 \%$ or more of the P-sites in-frame. In the case of multiple start codons, the most upstream in-frame start codon with a minimum of five P-sites in between it and the next ATG was selected. ORFs for which $>30 \%$ of the Ribo-Seq coverage was only supported by multimapping reads were also subsequently filtered. For a smORF to be considered actively translated in a condition, we required that it be identified in at least two of the three biological replicates for the condition.

Specific metaplots were also created for the 45 translated IncRNA-smORFs, and 100 randomly selected translated ccds ORFs, to compare ribosome enrichment around the start and stop codons in our protocol. P-sites were computed for each position in a 75 nt window around the start and stop codons, and scaled by the total number of reads in the two windows for each transcript. The mean normalized counts were then taken for each position in the two windows and plotted.

Translational efficiency (TE) was estimated for all translated ORFs in each condition, where TE was equal to the mean number 
of $\mathrm{P}$ sites per ORF, normalized by the median $\mathrm{P}$ sites per ORF per replicate, divided by the mean number of RNA sites per ORF, normalized by the median RNA sites per ORF per replicate.

\section{smORF peptide analysis}

For each of our ORF sets (protein coding, IncRNA-smORF, uORF, and dORFs), the average amino acid compositions were calculated. Random control expected frequencies were taken from King and Jukes (King and Jukes 1969).

Two published SH-SY5Y cell mass proteomics data sets were analyzed: PXD010776 (Murillo et al. 2018) and PXD014381 (Brenig et al. 2020). Binary raw files (*.raw) were downloaded from PRIDE then converted to human-readable MGF format using ThemoRawFileParser (Hulstaert et al. 2020). The amino acid sequences of our translated uORFs, dORFs, and IncRNA-smORFs were added to the whole Homo sapiens proteome data set (20,379 entries) downloaded from UniProtKB (Bateman et al. 2019) in November 2019. The new FASTA file was then used as a customized database on Comet (v2019.01.2) (Eng et al. 2013) search engine runs that scanned all MS/MS files (*.mgf) against it.

Default settings in Comet were used with the following exceptions according to the MS/MS data type. iTRAQ-4plex (PXD010776): decoy_search $=1$, peptide_mass_tolerance $=$ 10.00 , fragment_bin_tol $=0.1$, fragment_bin_offset $=0.0$, theoretical_fragment_ions $=0$, spectrum_batch_size $=15000$, clear_mz_ range $=113.5-117.5$, add_Nterm_peptide $=144.10253$, add_K_ lysine $=144.10253$, minimum_peaks $=8$. Label-free $($ PXD014381): decoy_search $=1$, peptide_mass_tolerance $=10.00$, fragment_ bin_tol $=0.02$, fragment_bin_offset $=0.0$, theoretical_fragment_ions $=0$, spectrum_batch_size $=15000$. CometUl (Eng et al. 2013) was used for analyzing MS/MS data and setting a false discovery rate (FDR) threshold of $10 \%$ per peptide identification. This FDR threshold was selected due to expected low abundance levels of the target smORFs.

\section{Conservation analysis}

Protein, cDNA, and ncRNA sequence data for $H$. sapiens, $P$. abelii, P. paniscus, P. troglodytes, G. gorilla, N. leucogenys, and M. musculus were obtained from Ensembl (release 100 [Yates et al. 2020]). LncRNAs are poorly conserved so we selected five species of apes with well-annotated genomes (four of these are great apes), and M. musculus represents an outgroup. These data formed the subject database for subsequent homology searches.

A number of criteria were considered to deem an ORF "conserved." At the protein level, these included a pairwise distance of $50 \%$ or less, syntenous positions in the genome, and the finding of ortholog groups exhibiting similar conservation to the human ORF. At the transcript level (using cDNA and ncRNA data), the above criteria were considered, along with the conservation of a start codon and subsequent sequence. If the same results were returned multiple times by the search strategies described below, they were also given extra consideration. In all cases the focus was on the conservation of the ORF sequence, not necessarily the surrounding transcript.

Sequence homology searches were performed using BLASTp (e-value $=0.001)$ where the 45 translated human IncRNA peptide sequences formed the queries and the protein sequences for P. abelii, P. paniscus, P. troglodytes, G. gorilla, N. leucogenys, and $M$. musculus formed the subject database (Altschul et al. 1990). Results were filtered to remove anything with $<75 \%$ identity, unless a result(s) was the lowest e-value hit for a given query in each species. Results were returned for 12 IncRNA peptides, and these were manually cross-validated using the Ensembl Genome Browser and multiple sequence alignments generated in ClustalOmega (Sievers et al. 2011). Default parameter settings were applied in the msa package in R (Bodenhofer et al. 2015).

The transcript sequences of the 45 translated IncRNAs were searched against transcriptome databases created by combining the cDNA and ncRNA data for each species, using BLASTn (e-val$\mathrm{ue}=0.001$ ) (Altschul et al. 1990). Results of this BLAST were used to filter the initial BLAST databases. ORF portions of the 45 translated IncRNAs were extracted and searched against these filtered databases using BLASTn (e-value =0.001) (Altschul et al. 1990).

The homology searches confirmed the genes of origin for all 45 IncRNA-smORFs in $\mathrm{H}$. sapiens at the nucleotide and peptide level. For the nucleotide sequence searches, the remaining species could identify homologs for 18 of the IncRNA ORF queries. These were cross-validated as described above (i.e., manually and using MSAs), resulting in 14 IncRNA-smORFs with evidence of sequence conservation based on transcript sequences. For the protein sequences, the remaining species returned result homologs for 21 of the IncRNA peptide queries. As some queries had many spurious results, they were further filtered to select the transcripts(s) with the lowest e-value for each query in each species. These were cross validated as above, resulting in 16 IncRNA peptides with evidence of sequence conservation based on transcript sequences. We combined evidence from both approaches into a final data set consisting of 17 IncRNA-smORFs with evidence of conservation in at least one of the six species queried.

The nucleotide alignments of the 17 IncRNA-smORFs were manually curated and trimmed, and evaluated using the 58 mammals model in PhyloCSF (Lin et al. 2011). As Bonobo is not in this model, three sequences could not be evaluated, and the putative Bonobo ORF was removed from a further three alignments.

\section{Cytoplasmic/nuclear fractionation of SH-SY5Y cells}

Cells were harvested and washed with 1X PBS. Cells were lysed in whole-cell lysis buffer (Supplemental Methods) (500 $\mu \mathrm{L}$ buffer per $10^{6}$ cells) on ice for $30 \mathrm{~min}$. Whole cell lysate aliquots were removed and remainder subjected to centrifugation at $1,600 \mathrm{~g}$ for $8 \mathrm{~min}$ to pellet nuclei. Nuclear and cytoplasmic fractions were subjected to two further clearing steps by centrifugation $(3000 \mathrm{~g}$ and $10,000 \mathrm{~g}$, respectively). Nuclei were lysed in RIPA buffer (Supplemental Methods). Approximately 10\% of both nuclear and cytoplasmic lysates were used for western blot and $~ 90 \%$ subjected to RNA extraction (ZYMO R1055).

\section{Western blot}

Samples were diluted in $4 \times$ Laemmli sample buffer (Bio-Rad) (277.8 mM Tris- $\mathrm{HCl}$, pH 6.8, 4.4\% LDS, 44.4\% (v/v) glycerol, $0.02 \%$ bromophenol blue), $5 \% \beta$-mercaptoethanol (Sigma) was added prior to heating at $95^{\circ} \mathrm{C}$ for $5 \mathrm{~min}$ and loaded on $10 \%$ SDS gels. Gel electrophoresis was performed using the Bio-Rad 
Mini-PROTEAN 3 gel electrophoresis system (Bio-Rad Laboratories). Proteins were transferred to nitrocellulose membranes (Amersham Protran) and blocked with $5 \%$ fat-free milk powder in $1 \times$ PBS, 0.05\% Tween-20 (Sigma) for $1 \mathrm{~h}$ at RT. Blots were incubated with primary antibodies overnight (Table 1). Blots were then washed in PBS-T and incubated with secondary antibody (anti-mouse HRP) at RT for $2 \mathrm{~h}$. Membranes were washed three times with PBS-T, prior to application of ECL (Biological Industries). Chemiluminescent signal was detected with ChemiDoc (Bio-Rad). All membranes were probed for $\beta$-tubulin as loading control.

\section{Analysis of publicly available IncRNA data}

Dynamic differential expression analysis data were accessed through IncExpDB (Cardoso-Moreira et al. 2019; Sarropoulos et al. 2019), where R package maSigPro was used to identify developmentally dynamically IncRNAs, that is, genes that show large changes in expression during development of a specific organ. Thirty-two IncRNA genes identified by Poly-Ribo-Seq as translated were present in IncExpDB. Disease association analysis used data from IncRNADisease (Chen et al. 2013; Bao et al. 2019), Differential Expression Atlas (https://www.ebi.ac.uk/gxa/ experiments?species=homo\%20sapiens), Cancer RNA-seq Nexus (Li et al. 2016), Malacards (Rappaport et al. 2013, 2014, 2017). Genes were considered related to a disease if they showed significant differential expression between diseased and control conditions $\left(\log _{2}\right.$ fold $\left.>1, P<0.05\right)$ or if they had been experimentally validated in the literature.

\section{smORF tagging}

5 '-UTRs and CDSs of putative smORFs (lacking stop codon) were generated by PCR (Supplemental Methods), using NEB High Fidelity DNA Polymerase (Q5). Carboxy-terminal 3×FLAG tag was incorporated within the reverse primer (Supplemental Methods) by PCR and products were cloned into Nhel and EcoRV restriction sites (Supplemental Methods) of pcDNA3.1/ Hygro Vector (Addgene, kindly provided by Mark RichardsBayliss group, University of Leeds). Start codon mutations were generated by site directed mutagenesis (Q5 Site Directed Mutagenesis Kit, NEB).

Plasmid transfections were performed using Lipofectamine 3000 (Thermo) following the manufacturer's instructions. After $48 \mathrm{~h}$, the cells were fixed for 20 min with $4 \%$ paraformaldehyde, washed with $1 \times$ PBS, $0.1 \%$ Triton X-100 (PBS-T) and processed for immunocytochemistry as previously described. Imaging was conducted using EVOS fluorescent microscope.

\section{siRNA knockdown}

siRNA knockdown was performed using Lincode siRNA SMARTpool (Dharmacon) (LINC01116 transcript: R-027999-000005 SMARTpool). Lincode Non-targeting Pool (D-001810-10) was used as scrambled control. Cells were seeded in 24-well plates $\left(10^{5}\right.$ cells/well, $)$ and siRNA were transfected using RNAiMAX lipofectamine (Thermo Fisher) as per manufacturer's instructions.

\section{General statistics and plots}

Statistical analyses were performed in R (R Core Team 2019), using packages including stringr (Wickham 2019), dplyr (Wickham et al. 2017), tidyr (Wickham 2017), protr (Xiao et al. 2015) ggplot2 (Wickham 2016), ggstatsplot (Patil 2018), knitr (Xie 2020), seqinr (Charif and Lobry 2007), ggbeeswarm (Clarke and Sherrill-Mix 2017), and EnhancedVolcano (Blighe et al. 2018).

Experimental values (RT-qPCR, are under polysome graphs, \% of cells) from independent samples with equal variances were assessed using two-tailed unpaired Student's t-test. The results are shown as mean \pm SEM values of three independent replicates. The exact $P$-values are described and specified in each figure legend. $P$-values $<0.05$ were considered statistically significant.

\section{DATA DEPOSITION}

Poly-Ribo-Seq data sets have been deposited in GEO with ID GSE166214.

\section{SUPPLEMENTAL MATERIAL}

Supplemental material is available for this article.

\section{ACKNOWLEDGMENTS}

We wish to thank Dr. Eric Hewitt for providing the SH-SY5Y cells; Dr. losifina Sampson and Dr. Mark Richards (Bayliss group) for providing HEK293 cells and mammalian vectors. We thank the Next Generation Sequencing facility at St. James University Hospital, Leeds, UK for performing Next Generation Sequencing. We also thank the Imaging Facility, Faculty of Biological Sciences, University of Leeds, UK for their assistance in confocal microscopy. Parts of this work were undertaken on ARC3, part of the High Performance Computing facilities at the University of Leeds, UK. This work was funded by the Medical Research Council (MRC; MR/N000471/1). K.A. was funded by Leeds Anniversary Research Scholarship (LARS). A.B.'s summer project was funded by White Rose BBSRC Doctoral Training Partnership in Mechanistic Biology (BB/M011151/1). A.K. is supported by a studentship from MRC Discovery Medicine North (DiMeN) Doctoral Training Partnership (MR/N013840/1). J.A. is funded by the University of Leeds (University Academic Fellow scheme).

Author contributions: K.D. designed and performed experiments, acquired, analyzed, and interpreted data, drafted and revised the manuscript. I.B. designed and performed experiments, acquired, analyzed, and interpreted data, drafted and revised the manuscript. D.W. analyzed, interpreted data, and revised the manuscript. A.K. analyzed, interpreted data, and revised the manuscript. S.C. acquired and analyzed data, and revised the manuscript. A.B. acquired and analyzed data, and revised the manuscript. E.J.R.V. analyzed data and revised the manuscript. M.O.C. helped interpret portions of the data and critique the output for important intellectual content, and revised the manuscript. J.D. helped design experiments, interpret portions of the data, and critique the output for important intellectual content, and revised the manuscript. A.W. helped design experiments, interpret portions of the data, and critique the output for important 
intellectual content, and revised the manuscript. J.L.A. conceived the work, interpreted data, drafted and revised the manuscript.

Received April 12, 2021; accepted June 22, 2021.

\section{REFERENCES}

Altschul SF, Gish W, Miller W, Myers EW, Lipman DJ. 1990. Basic local alignment search tool. J Mol Biol 215: 403-410. doi:10.1016/ S0022-2836(05)80360-2

Anderson DM, Anderson KM, Chang CL, Makarewich CA, Nelson BR, McAnally JR, Kasaragod P, Shelton JM, Liou J, Bassel-Duby R, et al. 2015. A micropeptide encoded by a putative long noncoding RNA regulates muscle performance. Cell 160: 595-606. doi:10.1016/j.cell.2015.01.009

Andrews S. 2010. FastQC: a quality control tool for high throughput sequence data. http://www.bioinformatics.babraham.ac.uk/projects/ fastqc.

Aspden JL, Eyre-Walker YC, Phillips RJ, Amin U, Mumtaz MA, Brocard M, Couso JP. 2014. Extensive translation of small open reading frames revealed by Poly-Ribo-Seq. Elife 3: e03528. doi:10.7554/eLife.03528

Bao Z, Yang Z, Huang Z, Zhou Y, Cui Q, Dong D. 2019. LncRNADisease 2.0: an updated database of long non-coding RNA-associated diseases. Nucleic Acids Res 47: D1034-D1037. doi:10.1093/nar/gky905

Bateman A, Martin MJ, Orchard S, Magrane M, Alpi E, Bely B, Bingley M, Britto R, Bursteinas B, Busiello G, et al. 2019. UniProt: a worldwide hub of protein knowledge. Nucleic Acids Res 47: D506-D515. doi:10.1093/nar/gky1049

Bazzini AA, Johnstone TG, Christiano R, Mackowiak SD, Obermayer B, Fleming ES, Vejnar CE, Lee MT, Rajewsky N, Walther TC, et al. 2014. Identification of small ORFs in vertebrates using ribosome footprinting and evolutionary conservation. EMBO J 33: 981993. doi:10.1002/embj.201488411

Blair JD, Hockemeyer D, Doudna JA, Bateup HS, Floor SN. 2017. Widespread translational remodeling during human neuronal differentiation. Cell Rep 21: 2005-2016. doi:10.1016/j.celrep.2017 .10 .095

Blighe K, Rana S, Lewis M. 2018. EnhancedVolcano: publication-ready volcano plots with enhanced colouring and labeling. https:// github.com/kevinblighe/EnhancedVolcano.

Bodenhofer U, Bonatesta E, Horejs-Kainrath C, Hochreiter S. 2015. msa: an R package for multiple sequence alignment. Bioinformatics 31: 3997-3999. doi:10.1093/bioinformatics/btv494

Brenig K, Grube L, Schwarzländer M, Köhrer K, Stühler K, Poschmann G. 2020. The proteomic landscape of cysteine oxidation that underpins retinoic acid-induced neuronal differentiation. J Proteome Res 19: 1923-1940. doi:10.1021/acs.jproteome $.9 \mathrm{~b} 00752$

Brockdorff N. 2018. Local tandem repeat expansion in Xist RNA as a model for the functionalisation of ncRNA. Non Coding RNA 4: 28. doi:10.3390/ncrna4040028

Brodie S, Lee HK, Jiang W, Cazacu S, Xiang C, Poisson LM, Datta I, Kalkanis S, Ginsberg D, Brodie C. 2017. The novel long non-coding RNA TALNEC2, regulates tumor cell growth and the stemness and radiation response of glioma stem cells. Oncotarget 8: 31785 31801. doi:10.18632/oncotarget.15991

Calviello L, Mukherjee N, Wyler E, Zauber H, Hirsekorn A, Selbach M, Landthaler M, Obermayer B, Ohler U. 2016. Detecting actively translated open reading frames in ribosome profiling data. Nat Methods 13: 165-170. doi:10.1038/nmeth.3688

Cardoso-Moreira M, Halbert J, Valloton D, Velten B, Chen C, Shao Y, Liechti A, Ascenção K, Rummel C, Ovchinnikova S, et al. 2019.
Gene expression across mammalian organ development. Nature 571: 505-509. doi:10.1038/s41586-019-1338-5

Carelli S, Giallongo T, Rey F, Latorre E, Bordoni M, Mazzucchelli S, Gorio MC, Pansarasa O, Provenzani A, Cereda C. 2019. HuR interacts with lincBRN1 $a$ and lincBRN1b during neuronal stem cells differentiation. RNA Biol 16: 1471-1485. doi:10.1080/15476286 .2019 .1637698

Carlevaro-Fita J, Rahim A, Guigó R, Vardy LA, Johnson R. 2016. Cytoplasmic long noncoding RNAs are frequently bound to and degraded at ribosomes in human cells. RNA 22: 867-882. doi:10.1261/rna.053561.115

Carrieri C, Cimatti L, Biagioli M, Beugnet A, Zucchelli S, Fedele S, Pesce E, Ferrer I, Collavin L, Santoro C, et al. 2012. Long non-coding antisense RNA controls Uchl1 translation through an embedded SINEB2 repeat. Nature 491: 454-457. doi:10.1038/ nature11508

Chan PP, Lowe TM. 2016. GtRNAdb 2.0: an expanded database of transfer RNA genes identified in complete and draft genomes. Nucleic Acids Res 44: D184-D189. doi:10.1093/nar/gkv1309

Charif D, Lobry JR. 2007. SeqinR 1.0-2: a contributed package to the R project for statistical computing devoted to biological sequences retrieval and analysis. In Structural approaches to sequence evolution: molecules networks populations (ed. Bastolla U, et al.), pp. 207-232. Springer, NY.

Chau KF, Shannon ML, Fame RM, Fonseca E, Mullan H, Johnson MB, Sendamarai AK, Springel MW, Laurent B, Lehtinen MK. 2018. Downregulation of ribosome biogenesis during early forebrain development. Elife 7: e36998. doi:10.7554/eLife.36998

Chen G, Wang Z, Wang D, Qiu C, Liu M, Chen X, Zhang Q, Yan G, Cui Q. 2013. LncRNADisease: a database for long-non-coding RNA-associated diseases. Nucleic Acids Res 41: D983-D986. doi:10.1093/nar/gks1099

Chen J, Brunner A-D, Cogan JZ, Nuez JK, Fields AP, Adamson B, Itzhak DN, Li JY, Mann M, Leonetti MD, et al. 2020. Pervasive functional translation of noncanonical human open reading frames. Science 367: 1140-1146. doi:10.1126/science.aay0262

Chodroff RA, Goodstadt L, Sirey TM, Oliver PL, Davies KE, Green ED, Molnár Z, Ponting CP. 2010. Long noncoding RNA genes: conservation of sequence and brain expression among diverse amniotes. Genome Biol 11: R72. doi:10.1186/gb-2010-11-7-r72

Chong C, Müller M, Pak H, Harnett D, Huber F, Grun D, Leleu M, Auger A, Arnaud M, Stevenson BJ, et al. 2020. Integrated proteogenomic deep sequencing and analytics accurately identify noncanonical peptides in tumor immunopeptidomes. Nat Commun 11: 1293. doi:10.1038/s41467-020-14968-9

Clarke E, Sherrill-Mix SC. 2017. ggbeeswarm: Categorical Scatter (Violin Point) Plots. https://cran.r-project.org/web/packages/ ggbeeswarm/index.html

Derrien T, Johnson R, Bussotti G, Tanzer A, Djebali S, Tilgner H, Guernec G, Martin D, Merkel A, Knowles DG, et al. 2012. The GENCODE v7 catalog of human long noncoding RNAs: analysis of their gene structure, evolution, and expression. Genome Res 22: 1775-1789. doi:10.1101/gr.132159.111

Dimartino D, Colantoni A, Ballarino M, Martone J, Mariani D, Danner J, Bruckmann A, Meister G, Morlando M, Bozzoni I. 2018. The long non-coding RNA Inc-31 interacts with Rock1 mRNA and mediates its YB-1-dependent translation. Cell Rep 23: 733-740. doi:10.1016/j.celrep.2018.03.101

Djebali S, Davis CA, Merkel A, Dobin A, Lassmann T, Mortazavi A, Tanzer A, Lagarde J, Lin W, Schlesinger F, et al. 2012. Landscape of transcription in human cells. Nature 489: 101-108. doi:10.1038/nature11233

Dobin A, Davis CA, Schlesinger F, Drenkow J, Zaleski C, Jha S, Batut P, Chaisson M, Gingeras TR. 2012. STAR: ultrafast universal RNA-seq 
aligner. Bioinformatics 29: 15-21. doi:10.1093/bioinformatics/ bts635

Duncan CDS, Mata J. 2014. The translational landscape of fissionyeast meiosis and sporulation. Nat Struct Mol Biol 21: 641-647. doi: $10.1038 / \mathrm{nsmb} .2843$

Eden E, Navon R, Steinfeld I, Lipson D, Yakhini Z. 2009. GOrilla: a tool for discovery and visualization of enriched $\mathrm{GO}$ terms in ranked gene lists. BMC Bioinformatics 10: 48. doi:10.1186/1471-2105-10-48

Eng JK, Jahan TA, Hoopmann MR. 2013. Comet: an open-source MS/ MS sequence database search tool. Proteomics 13: 22-24. doi:10 .1002/pmic.201200439

Faghihi MA, Zhang M, Huang J, Modarresi F, Van der Brug MP, Nalls MA, Cookson MR, St-Laurent G III, Wahlestedt C. 2010. Evidence for natural antisense transcript-mediated inhibition of microRNA function. Genome Biol 11: R56. doi:10.1186/gb2010-11-5-r56

Forster JI, Kglsberger S, Trefois C, Boyd O, Baumuratov AS, Buck L, Balling R, Antony PMA. 2016. Characterization of differentiated SH-SY5Y as neuronal screening model reveals increased oxidative vulnerability. J Biomol Screen 21: 496-509. doi:10.1177/ 1087057115625190

Frankish A, Diekhans M, Ferreira A-M, Johnson R, Jungreis I, Loveland J, Mudge JM, Sisu C, Wright J, Armstrong J, et al. 2019. GENCODE reference annotation for the human and mouse genomes. Nucleic Acids Res 47: D766-D773. doi:10.1093/nar/ gky955

Fujii K, Shi Z, Zhulyn O, Denans N, Barna M. 2017. Pervasive translational regulation of the cell signalling circuitry underlies mammalian development. Nat Commun 8: 14443. doi:10.1038/ ncomms 14443

Gordon A. 2010. FASTQ/A short-reads pre-processing tools. http ://hannonlab.cshl.edu/fastx_toolkit/.

Guo H, Ingolia NT, Weissman JS, Bartel DP. 2010. Mammalian microRNAs predominantly act to decrease target mRNA levels. Nature 466: 835-840. doi:10.1038/nature09267

Guttman M, Russell P, Ingolia NT, Weissman JS, Lander ES. 2013. Ribosome profiling provides evidence that large noncoding RNAs do not encode proteins. Cell 154: 240-251. doi:10.1016/j .cell.2013.06.009

Haeussler M, Zweig AS, Tyner C, Speir ML, Rosenbloom KR, Raney BJ, Lee CM, Lee BT, Hinrichs AS, Gonzalez JN, et al. 2019. The UCSC Genome Browser database: 2019 update. Nucleic Acids Res 47: D853-D858. doi:10.1093/nar/gky1095

Hannon GJ. 2010. FASTX-Toolkit. http://hannonlab.cshl.edu/ fastx_toolkit/

Hulstaert N, Shofstahl J, Sachsenberg T, Walzer M, Barsnes H, Martens L, Perez-Riverol Y. 2020. ThermoRawFileParser: modular, scalable, and cross-platform RAW file conversion. J Proteome Res 19: 537-542. doi:10.1021/acs.jproteome.9b00328

Ingolia NT, Brar GA, Rouskin S, McGeachy AM, Weissman JS. 2013. Genome-wide annotation and quantitation of translation by ribosome profiling. Curr Protoc Mol Biol 4: 4.18.11-14.18.19. doi:10 .1002/0471142727.mb0418s103

Johnsson P, Lipovich L, Grander D, Morris KV. 2014. Evolutionary conservation of long non-coding RNAs; sequence, structure, function. Biochim Biophys Acta 1840: 1063-1071. doi:10.1016/j.bbagen .2013.10.035

King JL, Jukes TH. 1969. Non-Darwinian evolution. Science 164: 788798. doi:10.1126/science.164.3881.788

Korecka JA, van Kesteren RE, Blaas E, Spitzer SO, Kamstra JH, Smit AB, Swaab DF, Verhaagen J, Bossers K. 2013. Phenotypic characterization of retinoic acid differentiated SH-SY5Y cells by transcriptional profiling. PLoS One 8: e63862. doi:10.1371/jour nal.pone.0063862
Kumar S, Stecher G, Suleski M, Hedges SB. 2017. TimeTree: a resource for timelines, timetrees, and divergence times. Mol Biol Evol 34: 1812-1819. doi:10.1093/molbev/msx116

Ladoukakis E, Pereira V, Magny EG, Eyre-Walker A, Couso JP. 2011. Hundreds of putatively functional small open reading frames in Drosophila. Genome Biol 12: 17. doi:10.1186/gb-2011-12-11r118

Langmead B, Salzberg SL. 2012. Fast gapped-read alignment with Bowtie 2. Nat Methods 9: 357-U354. doi:10.1038/nmeth.1923

Letunic I, Bork P. 2011. Interactive Tree Of Life v2: online annotation and display of phylogenetic trees made easy. Nucleic Acids Res 39: W475-W478. doi:10.1093/nar/gkr201

Li H, Handsaker B, Wysoker A, Fennell T, Ruan J, Homer N, Marth G, Abecasis G, Durbin R. 2009. The Sequence Alignment/Map format and SAMtools. Bioinformatics 25: 2078-2079. doi:10.1093/ bioinformatics/btp352

Li JR, Sun CH, Li W, Chao RF, Huang CC, Zhou XJ, Liu CC. 2016. Cancer RNA-Seq Nexus: a database of phenotype-specific transcriptome profiling in cancer cells. Nucleic Acids Res 44: D944D951. doi:10.1093/nar/gkv1282

Liao Y, Smyth GK, Shi W. 2013. The Subread aligner: fast, accurate and scalable read mapping by seed-and-vote. Nucleic Acids Res 41: e108. doi:10.1093/nar/gkt214

Liao Y, Smyth GK, Shi W. 2014. featureCounts: an efficient general purpose program for assigning sequence reads to genomic features. Bioinformatics 30: 923-930. doi:10.1093/bioinformatics/ btt656

Lin MF, Jungreis I, Kellis M. 2011. PhyloCSF: a comparative genomics method to distinguish protein coding and non-coding regions. Bioinformatics 27: i275-i282. doi:10.1093/bioinformatics/btr209

Lin N, Chang K-Y, Li Z, Gates K, Rana ZA, Dang J, Zhang D, Han T, Yang C-S, Cunningham TJ, et al. 2014. An evolutionarily conserved long noncoding RNA TUNA controls pluripotency and neural lineage commitment. Mol Cell 53: 1005-1019. doi:10.1016/j .molcel.2014.01.021

Lindsay SJ, Xu Y, Lisgo SN, Harkin LF, Copp AJ, Gerrelli D, Clowry GJ, Talbot A, Keogh MJ, Coxhead J, et al. 2016. HDBR expression: a unique resource for global and individual gene expression studies during early human brain development. Front Neuroanat 10: 86. doi:10.3389/fnana.2016.00086

Love MI, Huber W, Anders S. 2014. Moderated estimation of fold change and dispersion for RNA-seq data with DESeq2. Genome Biol 15: 550. doi:10.1186/s13059-014-0550-8

Magny EG, Pueyo JI, Pearl FM, Cespedes MA, Niven JE, Bishop SA, Couso JP. 2013. Conserved regulation of cardiac calcium uptake by peptides encoded in small open reading frames. Science 341: 1116-1120. doi:10.1126/science.1238802

Martin M. 2011. Cutadapt removes adapter sequences from highthroughput sequencing reads. EMBnet J 17: 3. doi:10.14806/ej 17.1.200

Michel AM, Mullan JPA, Velayudhan V, O'Connor PBF, Donohue CA, Baranov PV. 2016. RiboGalaxy: a browser based platform for the alignment, analysis and visualization of ribosome profiling data. RNA Biol 13: 316-319. doi:10.1080/15476286.2016.1141862

Murillo JR, Pla I, Goto-Silva L, Nogueira FCS, Domont GB, PerezRiverol Y, Sánchez A, Junqueira M. 2018. Mass spectrometry evaluation of a neuroblastoma SH-SY5Y cell culture protocol. Anal Biochem 559: 51-54. doi:10.1016/j.ab.2018.08.013

Patil I. 2018. ggstatsplot: 'ggplot2' based plots with statistical details. CRAN. https://indrajeetpatil.github.io/ggstatsplot/.

Patraquim P, Mumtaz MAS, Pueyo Jl, Aspden JL, Couso JP. 2020. Developmental regulation of canonical and small ORF translation from mRNAs. Genome Biol 21: 128. doi:10.1186/s13059-02002011-5 
Pedersen AG, Nielsen H. 1997. Neural network prediction of translation initiation sites in eukaryotes: perspectives for EST and genome analysis. Proc Int Conf Intell Syst Mol Biol 5: 226-233.

Ponting CP, Oliver PL, Reik W. 2009. Evolution and functions of long noncoding RNAs. Cell 136: 629-641. doi:10.1016/j.cell.2009.02 .006

Pueyo JI, Couso JP. 2008. The 11-aminoacid long Tarsal-less peptides trigger a cell signal in Drosophila leg development. Dev Biol 324: 192-201. doi:10.1016/j.ydbio.2008.08.025

Ramilowski JA, Yip CW, Agrawal S, Chang JC, Ciani Y, Kulakovskiy IV, Mendez M, Ooi JLC, Ouyang JF, Parkinson N, et al. 2020. Functional annotation of human long noncoding RNAs via molecular phenotyping. Genome Res 30: 1060-1072. doi:10.1101/gr .254219 .119

Rappaport N, Nativ N, Stelzer G, Twik M, Guan-Golan Y, Stein TI, Bahir I, Belinky F, Morrey CP, Safran M, et al. 2013. MalaCards: an integrated compendium for diseases and their annotation. Database (Oxford) 2013: bat018. doi:10.1093/database/bat018

Rappaport N, Twik M, Nativ N, Stelzer G, Bahir I, Stein TI, Safran M, Lancet D. 2014. MalaCards: a comprehensive automaticallymined database of human diseases. Curr Protoc Bioinformatics 47: 1.24.21-19. doi:10.1002/0471250953.bi0124s47

Rappaport N, Twik M, Plaschkes I, Nudel R, Iny Stein T, Levitt J, Gershoni M, Morrey CP, Safran M, Lancet D. 2017. MalaCards: an amalgamated human disease compendium with diverse clinical and genetic annotation and structured search. Nucleic Acids Res 45: D877-D887. doi:10.1093/nar/gkw1012

$R$ Core Team. 2019. $R$ : a language and environment for statistical computing. R Foundation for Statistical Computing, Vienna, Austria. https://www.R-project.org/

Rodriguez CM, Chun SY, Mills RE, Todd PK. 2019. Translation of upstream open reading frames in a model of neuronal differentiation. BMC Genomics 20: 391. doi:10.1186/s12864-019-5775-1

Ruiz-Orera J, Alba MM. 2019. Conserved regions in long non-coding RNAs contain abundant translation and protein-RNA interaction signatures. NAR Genomics Bioinformatics 1: e2. doi:10.1093/nar $\mathrm{gab} / \mathrm{lqz} 002$

Saghatelian A, Couso JP. 2015. Discovery and characterization of smORF-encoded bioactive polypeptides. Nat Chem Biol 11: 909-916. doi:10.1038/nchembio.1964

Sarropoulos I, Marin R, Cardoso-Moreira M, Kaessmann H. 2019. Developmental dynamics of IncRNAs across mammalian organs and species. Nature 571: 510-514. doi:10.1038/s41586-019$1341-x$

Shen S, Lin L, Cai JJ, Jiang P, Kenkel EJ, Stroik MR, Sato S, Davidson BL, Xing Y. 2011. Widespread establishment and regulatory impact of Alu exons in human genes. Proc Natl Acad Sci 108: 2837-2842. doi:10.1073/pnas.1012834108

Sievers F, Wilm A, Dineen D, Gibson TJ, Karplus K, Li WZ, Lopez R, McWilliam H, Remmert M, Soding J, et al. 2011. Fast, scalable generation of high-quality protein multiple sequence alignments using Clustal Omega. Mol Syst Biol 7: 6. doi:10 .1038/msb.2011.75

Spencer HL, Sanders R, Boulberdaa M, Meloni M, Cochrane A, Spiroski A-M, Mountford J, Emanueli C, Caporali A, Brittan M, et al. 2020. The LINC00961 transcript and its encoded micropep- tide, small regulatory polypeptide of amino acid response, regulate endothelial cell function. Cardiovasc Res 116: 1981-1994. doi:10.1093/cvr/cvaa008

Su X, Zhang J, Luo X, Yang W, Liu Y, Liu Y, Shan Z. 2019. LncRNA LINC01116 promotes cancer cell proliferation, migration and invasion in gastric cancer by positively interacting with IncRNA CASC11. Onco Targets Ther 12: 8117-8123. doi:10.2147/OTT .S208133

Szafron LM, Balcerak A, Grzybowska EA, Pienkowska-Grela B, FelisiakGolabek A, Podgorska A, Kulesza M, Nowak N, Pomorski P, Wysocki J, et al. 2015. The novel gene CRNDE encodes a nuclear peptide (CRNDEP) which is overexpressed in highly proliferating tissues. PLoS One 10: e0127475. doi:10.1371/journal.pone .0127475

Tsagakis I, Douka K, Birds I, Aspden JL. 2020. Long non-coding RNAs in development and disease: conservation to mechanisms. J Pathol 250: 480-495. doi:10.1002/path.5405

van Heesch S, Witte F, Schneider-Lunitz V, Schulz JF, Adami E, Faber AB, Kirchner M, Maatz H, Blachut S, Sandmann CL, et al. 2019. The translational landscape of the human heart. Cell 178: 242-260.e229. doi:10.1016/j.cell.2019.05.010

Wang H, lacoangeli A, Popp S, Muslimov IA, Hiroaki I, Sonenberg N, Lomakin IB, Tiedge H. 2002. Dendritic BC1 RNA: functional role in regulation of translation initiation. J Neurosci 22: 10232-10241. doi:10.1523/JNEUROSCI.22-23-10232.2002

Wang L, Fan J, Han L, Qi H, Wang Y, Wang H, Chen S, Du L, Li S, Zhang Y, et al. 2020. The micropeptide LEMP plays an evolutionarily conserved role in myogenesis. Cell Death Dis 11: 357 . doi:10 .1038/s41419-020-2570-5

Waterhouse AM, Procter JB, Martin DMA, Clamp M, Barton GJ. 2009. Jalview Version 2-a multiple sequence alignment editor and analysis workbench. Bioinformatics 25: 1189-1191. doi:10.1093/ bioinformatics/btp033

Wickham H. 2016. ggplot2: elegant graphics for data analysis. Springer-Verlag, New York.

Wickham H. 2017. tidyr: tidy messy data. R package version 0.7.2. https://CRAN.R-project.org/package=tidyr

Wickham H, François R, Henry L, Müller K. 2017. dplyr: a grammar of data manipulation. $\mathrm{R}$ package version 0.7.4. https://CRAN.Rproject.org/package $=$ dplyr

Wickham H. 2019. stringr: simple, consistent wrappers for common string operations. https://CRAN.R-project.org/package=stringr

Winzi MA. 2018. The long noncoding RNA IncR492 inhibits neural differentiation of murine embryonic stem cells. PLoS One 13: e0191682. doi:10.1371/journal.pone.0191682

Xiao N, Cao DS, Zhu MF, Xu OS. 2015. protr/ProtrWeb: R package and web server for generating various numerical representation schemes of protein sequences. Bioinformatics 31: 1857-1859. doi:10.1093/bioinformatics/btv042

Xie Y. 2020. knitr: a general-purpose package for dynamic report generation in $R$. https://yihui.org/knitr/

Yates AD, Achuthan P, Akanni W, Allen J, Alvarez-Jarreta J, Amode MR, Armean IM, Azov AG, Bennett R, Bhai J, et al. 2020. Ensembl 2020. Nucleic Acids Res 48: D682-D688. doi:10.1093/ nar/gkz1138 

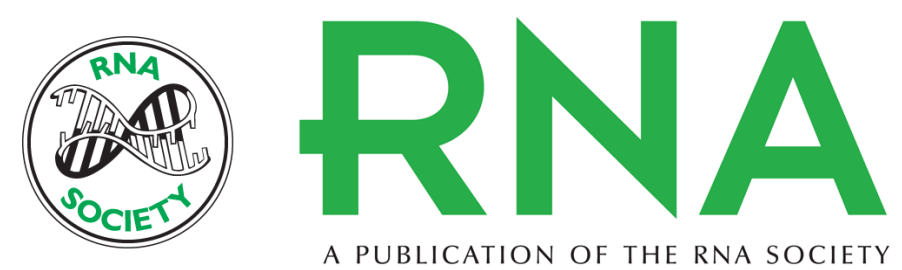

\section{Cytoplasmic long noncoding RNAs are differentially regulated and translated during human neuronal differentiation}

Katerina Douka, Isabel Birds, Dapeng Wang, et al.

RNA 2021 27: 1082-1101 originally published online June 30, 2021

Access the most recent version at doi:10.1261/rna.078782.121

Supplemental
Material http://rnajournal.cshlp.org/content/suppl/2021/06/30/rna.078782.121.DC1

References This article cites 82 articles, 8 of which can be accessed free at: http://rnajournal.cshlp.org/content/27/9/1082.full.html\#ref-list-1

Open Access Freely available online through the RNA Open Access option.

Creative This article, published in $R N A$, is available under a Creative Commons License Commons (Attribution 4.0 International), as described at

License http://creativecommons.org/licenses/by/4.0/.

Email Alerting Receive free email alerts when new articles cite this article - sign up in the box at the Service top right corner of the article or click here.

To subscribe to RNA go to:

http://rnajournal.cshlp.org/subscriptions

(C) 2021 Douka et al.; Published by Cold Spring Harbor Laboratory Press for the RNA Society 